bioRxiv preprint doi: https://doi.org/10.1101/2020.05.20.103614; this version posted May 21,2020 . The copyright holder for this preprint (which was not certified by peer review) is the author/funder, who has granted bioRxiv a license to display the preprint in perpetuity. It is made available under aCC-BY-NC-ND 4.0 International license.

Manuscript

Online article

Confidential (1) 1

\title{
Massively parallel quantification of CRISPR editing in cells by TRAP-seq enables better design of Cas9, ABE, CBE gRNAs of high efficiency and accuracy
}

\section{Authors}

Xi Xiang ${ }^{1-4}$ *, Kunli Qu ${ }^{1,5 *}$, Xue Liang ${ }^{1,5 *}$, Xiaoguang Pan ${ }^{1,5 *}$, Jun Wang ${ }^{1-3}$, Peng Han ${ }^{1,2,5}$, Zhanying Dong ${ }^{1}$, Lijun Liu ${ }^{1,5}$, Jiayan Zhong ${ }^{6}$, Tao Ma ${ }^{6}$, Yiqing Wang ${ }^{1}$, Jiaying $\mathrm{Yu}^{1,2}$, Xiaoying Zhao ${ }^{1,2}$, Siyuan $\mathrm{Li}^{1,2}$, Zhe $\mathrm{Xu}^{1,2}$, Jinbao Wang ${ }^{6}$, Xiuqing Zhang ${ }^{2,3}$, Hui Jiang ${ }^{6}$, Fengping Xu ${ }^{1,3}$, Lijin Zou ${ }^{7}$, Huajing Teng ${ }^{8}$, Xin Liu ${ }^{3}$, Xun $\mathrm{Xu}^{3,9}$, Jian Wang ${ }^{3}$, Huanming Yang ${ }^{3,10}$, Lars Bolund 1,3,4,5, George M. Church ${ }^{11}$, Lin Lin ${ }^{1,4,12, \$}$ \& Yonglun Luo ${ }^{1,3,4,5,12, \$, \#}$

\section{Affiliations}

${ }^{1}$ Lars Bolund Institute of Regenerative Medicine, BGI-Qingdao, Qingdao 266555, China.

${ }^{2}$ BGI Education Center, University of Chinese Academy of Sciences, Shenzhen 518083, China.

${ }^{3}$ BGI-Shenzhen, Shenzhen 518083, China.

${ }^{4}$ Department of Biomedicine, Aarhus University, Aarhus 8000, Denmark.

${ }^{5}$ Qingdao-Europe Advanced Institute for Life Sciences, BGI-Shenzhen, Qingdao 266555, China ${ }^{6}$ MGI, BGI-Shenzhen, Shenzhen 518083, China.

${ }^{7}$ The First Affiliated Hospital of Nanchang University, Nanchang, Jiangxi 330006, P.R. China ${ }^{8}$ Key Laboratory of Carcinogenesis and Translational Research, Department of Radiation Oncology, Peking University Cancer Hospital \& Institute, Beijing, China

${ }^{9}$ Guangdong Provincial Key Laboratory of Genome Read and Write, BGI-Shenzhen, Shenzhen, 518120, China

${ }^{10}$ Guangdong Provincial Academician Workstation of BGI Synthetic Genomics, BGI-Shenzhen, Shenzhen, 518120, China

${ }^{11}$ Department of Genetics, Blavatnik Institute, Harvard Medical School, Boston, MA 02115, USA

${ }^{12}$ Steno Diabetes Center Aarhus, Aarhus University, Aarhus 8200, Denmark \# lead contact

*These authors contribute equally to the study and should be regarded as co-first authors.

†Correspondence addressed to: Lin Lin: lin.lin@biomed.au.dk or Yonglun Luo: alun@biomed.au.dk 
bioRxiv preprint doi: https://doi.org/10.1101/2020.05.20.103614; this version posted May $21,2020$. The copyright holder for this preprint (which was not certified by peer review) is the author/funder, who has granted bioRxiv a license to display the preprint in perpetuity. It is made available under aCC-BY-NC-ND 4.0 International license.

\section{Manuscript}

Online article

Confidential

32

33

34

35

36

37

\section{Abstract}

The CRISPR RNA-guided endonucleases Cas9, and Cas9-derived adenine/cytosine base editors $(\mathrm{ABE} / \mathrm{CBE})$, have been used in both research and therapeutic applications. However, broader use of this gene editing toolbox is hampered by the great variability of efficiency among different target sites. Here we present TRAP-seq, a versatile and scalable approach in which the CRISPR gRNA expression cassette and the corresponding surrogate site are captured by Targeted Reporter Anchored Positional Sequencing in cells. TRAP-seq can faithfully recapitulate the CRISPR gene editing outcomes introduced to the corresponding endogenous genome site and most importantly enables massively parallel quantification of CRISPR gene editing in cells. We demonstrate the utility of this technology for high-throughput quantification of SpCas9 editing efficiency and indel outcomes for 12,000 gRNAs in human embryonic kidney cells. Using this approach, we also showed that TRAPseq enables high throughput quantification of both ABE and CBE efficiency at 12,000 sites in cells. This rich amount of $\mathrm{ABE} / \mathrm{CBE}$ outcome data enable us to reveal several novel nucleotide features (e.g. preference of flanking bases, nucleotide motifs, STOP recoding types) affecting base editing efficiency, as well as designing improved machine learning-based prediction tools for designing SpCas9, ABE and CBE gRNAs of high efficiency and accuracy ( $>70 \%)$. We have integrated all the 12,000 CRISPR gene editing outcomes for SpCas9, ABE and CBE into a CRISPR-centered portal: The Human CRISPR Atlas. This study extends our knowledge on CRISPR gene and base editing, and will facilitate the application and development of CRISPR in both research and therapy.

\section{Keywords}

Gene editing, A-to-G base editing, C-to-T base editing, Genome engineering, System biology 
bioRxiv preprint doi: https://doi.org/10.1101/2020.05.20.103614; this version posted May $21,2020$. The copyright holder for this preprint (which was not certified by peer review) is the author/funder, who has granted bioRxiv a license to display the preprint in perpetuity. It is made available under aCC-BY-NC-ND 4.0 International license.

\section{Manuscript}

Online article

Confidential

\section{INTRODUCTION}

Clustered Regularly Interspaced Short Palindromic Repeats (CRISPR) and CRISPR-associated protein 9 (Cas9) are essential adaptive immune components in most bacteria. The system has successfully been harnessed for programmable RNA-guided genome editing in prokaryotes, humans and many other living organisms [1-5]. The Streptococcus pyogenes Cas9 (SpCas9) is the most extensively studied and broadly applied Cas9 protein, amongst other Cas9 orthologs (e.g. SaCas9, StCas9, NmCas9) [6-9] and Cas proteins (e.g. Cas12a, Cas13) [10, 11]. Guided by a programmable small RNA molecule (also known as gRNA), the SpCas9 protein introduces a double-stranded DNA break (DSB) to the DNA target site, which constitutes a complementary protospacer sequences and a canonical protospacer adjacent motif (PAM) [2]. The classical CRISPR gene editing is achieved by reparation of the DSBs in living organisms by the endogenous DNA repair mechanisms, predominantly by the NHEJ and MMEJ pathways in mammalian cells. This process generates indels (deletions or insertions) to the repaired site [12]. It is thus essential to have data from CRISPR editing in cells to develop accurate prediction rule sets of CRISPR activity.

The simplicity of the CRISPR system, the flexibility for modifying the Cas 9 protein, and the increasing efforts from CRISPR scientists and pharmaceutical companies have extensively broadened the CRISPR-Cas9-based gene editing toolkits. We are now enabled to epigenetically perturb endogenous gene expression [13, 14], fluorescently label endogenous DNA elements[15] and sitespecifically edit single nucleotides [16-20]. The CRISPR base editors, which comprise two major classes: adenine base editors (ABE) and cytosine base editors (CBE), have increasingly evolved as attracting tools for gene editing. These base editors are created by fusing a catalytically dead Cas 9 (dCas9) or Cas9 nickase (nCas9) to either an adenine deaminase or a cytidine deaminase [18, 19]. Without introducing double stranded DNA breaks, the ABE and CBE base editors, respectively, can efficiently create an $\mathrm{A}$ to $\mathrm{G}$ (or $\mathrm{T}$ to $\mathrm{C}$ on the complementary strand) and $\mathrm{C}$ to $\mathrm{T}$ (or $\mathrm{G}$ to $\mathrm{A}$ on the complementary strand) substitution within a small editing window of the target site [16, 21-23]. Albeit all these fantastic developments and applications of the CRISPR-Cas9 gene editing theme, there is still an urgent need of methods and high throughput data on the Cas9-induced DBS repair outcomes, as well as ABE and CBE efficiencies, to ensure a successful CRISPR gene editing outcome. Such cataloged data of Cas9 and base editor efficiencies will allow the selection of experimentally validated gRNAs, as well as for developing better rules for in silico Cas9, ABE, and CBE gRNAs design. 
bioRxiv preprint doi: https://doi.org/10.1101/2020.05.20.103614; this version posted May 21, 2020. The copyright holder for this preprint (which was not certified by peer review) is the author/funder, who has granted bioRxiv a license to display the preprint in perpetuity. It is made available under aCC-BY-NC-ND 4.0 International license.

\section{Manuscript}

Online article

Confidential

89 Quantification of gRNA activity at the endogenous sites in cells is limited by scale. In vitro 90 approaches (in a test-tube) can overcome the scale but fails to recapitulate the effects of genome and 91 epigenome architectures and cellular DNA repair mechanisms on CRISPR editing [24, 25]. Methods 92 based on integrating synthetically barcoded DNA constructions were developed for large-scale 93 measuring of Cas9-induced DSB repair outcomes of gRNAs in cells [26-28]. Currently, we lack 94 large-scale $\mathrm{ABE}$ and $\mathrm{CBE}$ editing data for developing better rules for designing base editing gRNAs. 95 In this study, we developed an assay system for massively parallel quantification of a large-scale 96 CRISPR gRNAs activities in human cells. We optimized the design and procedures for generation 97 and in-cell CRISPR editing of synthetically barcoded DNA constructs. Each construct contains a 98 unique gRNA expression cassette and the corresponding surrogate target site. Using this method, 99 Targeted Reporter Anchored Positional Sequencing (hereafter referred as TRAP-seq), we 100 demonstrated the applicability of TRAP-seq for massively parallel quantification of the SpCas9101 induced DSB repair outcomes, ABE and CBE efficiency and profiles for 12,000 gRNAs in human 102 embryonic kidney cells. 
bioRxiv preprint doi: https://doi.org/10.1101/2020.05.20.103614; this version posted May $21,2020$. The copyright holder for this preprint (which was not certified by peer review) is the author/funder, who has granted bioRxiv a license to display the preprint in perpetuity. It is made available under aCC-BY-NC-ND 4.0 International license.

\section{Manuscript}

Online article

Confidential

105

106

107

108

109

110

111

112

113

114

115

116

117

118

119

120

121

122

123

124

125

126

127

\section{RESULTS}

\section{Design and functional validation of the lentivirus-based TRAP-seq system}

To streamline vector cloning, gRNA expression and delivery into cells, we firstly designed a lentivirus-based system with four main features: (1) A human U6 promoter; (2) Golden-Gate Assembly (GGA) based cloning with a lac $Z$ marker for precise and efficient insertion of an expression cassette; (3) A green fluorescent protein (GFP) marker for measuring viral titer and realtime tracking of viral delivery; (4) A puromycin selection gene for enrichment of stably transduced cells (Fig. 1a and S1). Essentially, this lentivirus system allows conventional GGA-based insertion of a synthetic DNA containing a gRNA spacer, scaffold and the corresponding surrogate target site after the U6 promoter. As current microarray-based method can only faithfully synthesize oligo pools of max $170 \mathrm{bp}$, we optimized the DNA design to contain a 102bp gRNA expression cassette (20bp spacer $+82 \mathrm{bp}$ scaffold) and a $37 \mathrm{bp}$ surrogate target site, flanked by a $31 \mathrm{bp}$ GGA cloning site and PCR handles (Fig. 1a and S1). We and several other groups previously demonstrated that such a surrogate target site can faithfully recapitulate the endogenous editing efficiency and indel profile [27, 29]. To further validate the $37 \mathrm{bp}$ surrogate target site, we firstly generated HEK293T cells expressing a doxycycline (Dox)-inducible SpCas9 [1], an adenine base editor (ABE7.10) [19] or a cytosine base editor (CBE, BE4-Gam) [30]. Next, we performed ICE-based analysis of three different sites (AAVS1, INHCB, TYMP) in the HEK293T-SpCas9, HEK293T-ABE and HEK293T-CBE cells. The results validated that the CRISPR editing efficiency and outcomes from the surrogate sites were closely correlated $\left(r^{2}=\mathbf{0 . 9 6}-\mathbf{0 . 9 9}\right)$ with those from the endogenous genome sites (Fig. S2). For simplification, we hereafter named the system as Targeted Reporter Anchored Positional Sequencing (TRAP-seq), the 170bp synthetic oligo/DNA as TRAP oligo/DNA, and the 37bp surrogate target site as TRAP site. 
bioRxiv preprint doi: https://doi.org/10.1101/2020.05.20.103614; this version posted May 21, 2020. The copyright holder for this preprint (which was not certified by peer review) is the author/funder, who has granted bioRxiv a license to display the preprint in perpetuity. It is made available under aCC-BY-NC-ND 4.0 International license.

Manuscript Online article

a

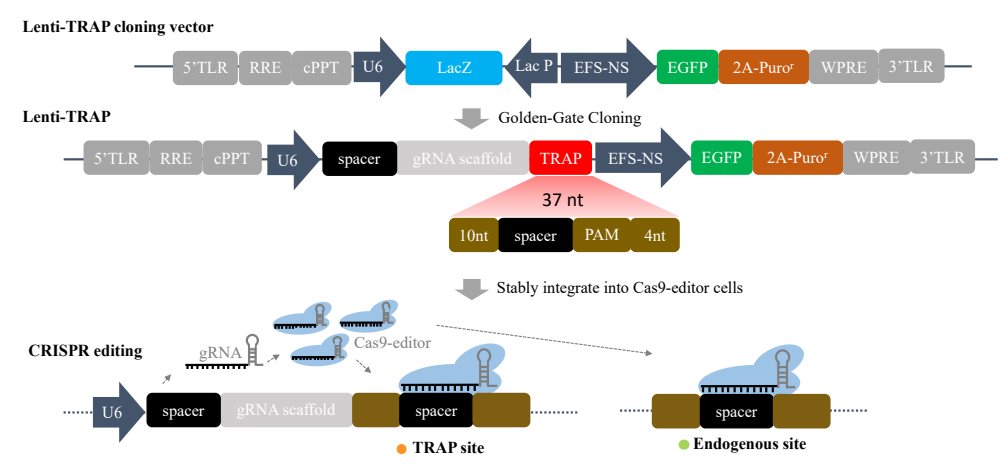

b

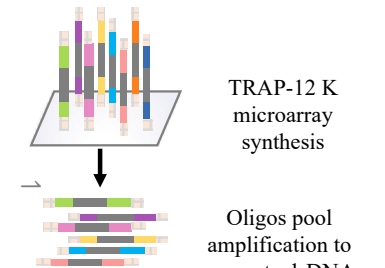
generate dsDNA
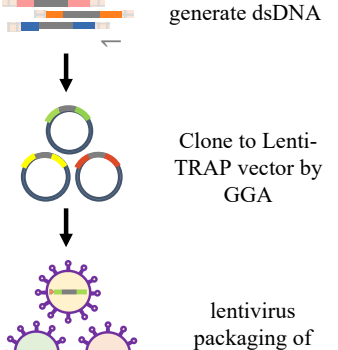

lentivirus 年 $\downarrow$ d
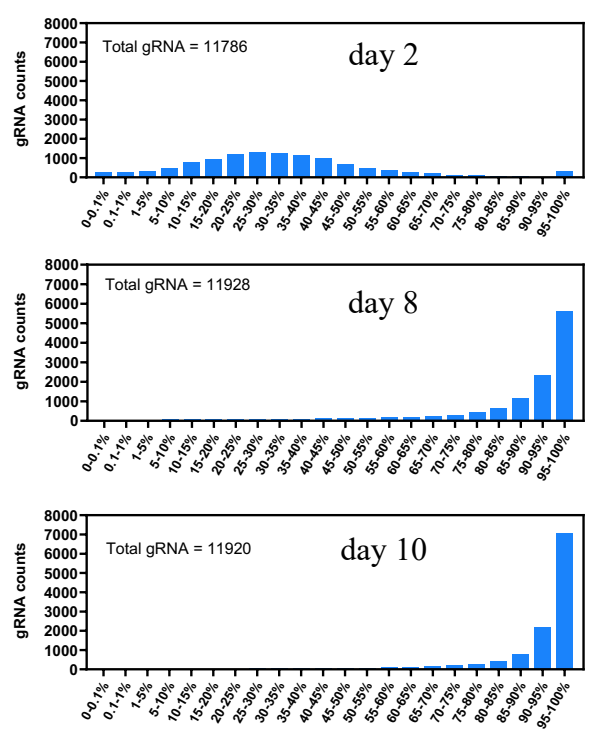

Transduce DOX

Cas-editor cells

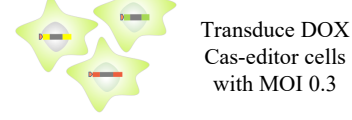
to scale. transduction of the 12K TRAP-seq library. 12,000 TRAP sites can be found at the CRISPR atlas database. Dox-free HEK293T-SpCas9 are shown in Fig. S10.
Confidential

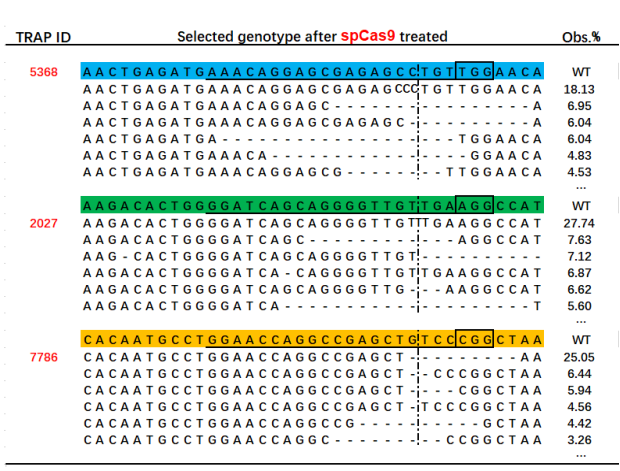

e
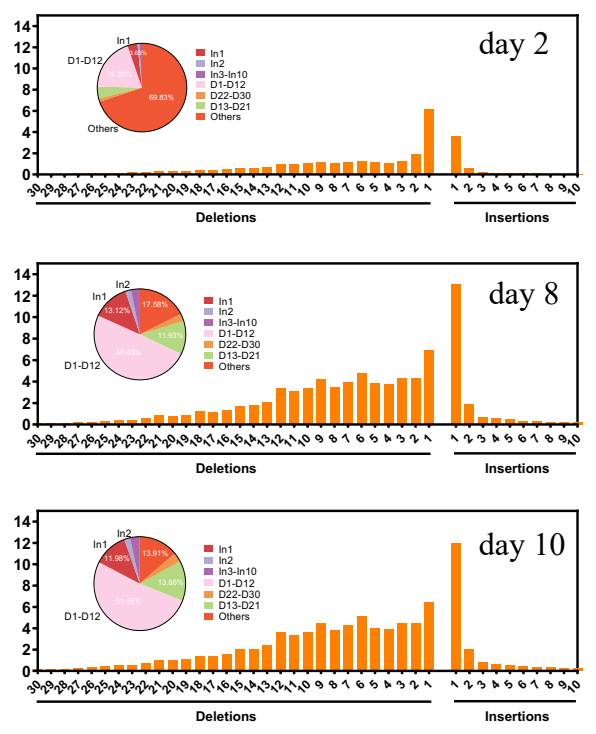

\section{Figure 1 High Throughput Quantification of SpCas9 efficiency in cells by TRAP-seq}

a. Schematic illustration of the TRAP-seq system. TLR, long terminal repeat; RRE, Rev Response Element; cPPT, central polypurine tract; U6, human U6 promoter; EFS-NS, short EFS promoter derived from EF1a. Figure not drown

b. Schematic illustration of oligo pool synthesis, PCR amplification, gold-gate assembly, lentivirus packaging, and

c. Representative quantification of top 5 indel types for 3 TRAP sites. Dash line indicates the DSB site. Results for the

d. Bar plots of SpCas9 editing efficiency of all TRAP sites measured by targeted amplicon sequencing. Results are shown for Dox-induced HEK293T-SpCas9 cells from 2, 8 and 10 days after transduction. Corresponding results for

e. Bar plots of indel profiles (1-20 bp deletion, 1-10 bp insertion) for all TRAP sites introduced by SpCas9 in the Doxinduced HEK293T-SpCas9 cells from at 2, 8, and 10 days post transduction. Pie chat quantified the proportion of major indel groups: $1 \mathrm{bp}$ insertion (ins), 2bp insertion, 3-10 bp insertion, 1-12 bp deletion, 13-21bp deletion and 22-30 bp 
bioRxiv preprint doi: https://doi.org/10.1101/2020.05.20.103614; this version posted May $21,2020$. The copyright holder for this preprint (which was not certified by peer review) is the author/funder, who has granted bioRxiv a license to display the preprint in perpetuity. It is made available under aCC-BY-NC-ND 4.0 International license.

\section{Manuscript}

Online article

Confidential

deletion. Other indels and wild-type reads are presented together as "others". Corresponding results for Dox-free HEK293T-SpCas9 are shown in Fig. S11 and S12.

\section{Generation of 12K TRAP-seq lentiviral library}

We next generated a 12K TRAP-seq library comprising 12,000 TRAP oligos by microarray synthesis (Fig. 1b). The library targets 3,834 human protein-coding genes (Table S1) [31]. The gRNA spacers were selected from the iSTOP database [32]. Out optimized workflow (also seen in methods) for PCR amplification of the 12K TRAP-seq oligos and cloning into the lentivirus-based TRAP-seq vector system is illustrated in Fig. S3a. A serial of optimizations in PCR conditions, GGA reactions and lentiviral packaging were carried out to avoid unspecific amplification (Fig. S3b, c), maximize successful ligation (Fig. S4) and properly quantify viral titer by FACS (Fig. S5), respectively.

To analyze the coverage of each TRAP oligo in our 12K TRAP-seq library, as well as to assess if the whole procedure of vector cloning and lentivirus packaging/transduction affected the overall TRAP representation, we performed targeted amplicon sequencing of the TRAP DNA in the 12K TRAPseq oligo library, GGA plasmid DNA and wildtype HEK293T cells transduced with the 12K TRAPseq lentivirus with a multiplexity of infection (MOI) of 0.3 . With a constant sequencing depth ( $>$ 1,000X), all 12,000 TRAP oligos were detected in the 12K TRAP-seq library and the majority of TRAP oligos (>90\%) were evenly distributed (Fig. S6a). Most importantly, over 99\% of the TRAP oligos were present in the $12 \mathrm{~K}$ TRAP-seq plasmids and lentivirus preparation with high correlation of representation for each TRAP oligo $\left(\mathbf{r}^{2}=\mathbf{0 . 8 6 - 0 . 9 1}\right.$, Fig. S6b), suggesting that our optimized PCR, GGA, lentivirus packaging and transduction methods faithfully retained the complexity of the $12 \mathrm{~K}$ TRAP-seq library without causing dramatic over/under-representation of the TRAP oligos.

\section{Quantification of SpCas9 editing at 12,000 sites by TRAP-seq}

To demonstrate applicability of the 12K TRAP-seq lentivirus library, we firstly investigated massively parallel quantification of SpCas9 editing activity at all 12,000 TRAP sites. As schematically shown in Fig. S7, we transduced the Dox inducible HEK293T-SpCas9 cells with the 12K TRAP-seq lentivirus ( $\mathrm{MOI}=0.3$ and transduction coverage $=4,690$ cells per TRAP). Puromycin selection and Dox addition started two days after transduction to achieve maximum transduction and gene editing efficiency (Fig. S8). To enable comparison and identification of CRISPR-introduced indels, we also transduced wildtype HEK293T cells with the 12K TRAP-seq lentivirus with same MOI and transduction coverage. We harvested genomic DNA from the transduced cells at three time points: 2, 8, and 10 days after transduction (Fig. S7 and Fig. S8). Targeted PCRs were performed 
bioRxiv preprint doi: https://doi.org/10.1101/2020.05.20.103614; this version posted May $21,2020$. The copyright holder for this preprint (which was not certified by peer review) is the author/funder, who has granted bioRxiv a license to display the preprint in perpetuity. It is made available under aCC-BY-NC-ND 4.0 International license.

\section{Manuscript}

Online article

Confidential

with a pair of universal primers specifically amplifying the TRAP DNA, followed by targeted deep sequencing with a DNA Nanoball sequencing technology [33]. With a constant sequencing depth (Fig S9), the proportional representation of each TRAP correlated better in the Dox-free HEK293TSpCas 9 cells $(\mathbf{r}=\mathbf{0 . 9 5})$ than that in the Dox-induced HEK293T-SpCas9 cells $(\mathbf{R}=\mathbf{0 . 8 8})$. Similar to CRISPR knockout screening pool libraries [34, 35], our results suggested that there existed similar cell fitness-related enrichment and depletion of the gRNAs in the 12K TRAP-seq library.

To measure the SpCas9 editing outcome, we firstly filtered out indels commonly found in both WT and SpCas9 HEK293T cells (also see methods), which were introduced by oligo synthesis or PCRs. We next analyzed the editing frequency and indel profiles for all 12,000 TRAP sites (Fig. 1c, Table S2, also see CRISPR Atlas below). Although the SpCas9 expression was Dox inducible, significant editing efficiencies were detected for all gRNAs in the HEK293T-SpCas9 cells at 2, 8 and 10 days after transduction in Dox-free medium (Fig. S10), suggesting a substantial leakiness of SpCas9 expression. As expected, significantly higher editing efficiencies were achieved for all 12,000 gRNAs in Dox-addition HEK293T-SpCas9 cells at 8 and 10 days after transduction (Fig. 1d). These results support the notion that SpCas9 expression level and cultivation time affect gene editing efficiency [36]. We and others had demonstrated that the indel outcomes introduced by SpCas9 comprises mainly small deletions and insertions [37-39]. The distribution of indel profiles (deletion of 1-30 bp and insertion of 1-10 bp) of the 12K TRAP sites were thus assessed in the transduced HEK293TSpCas 9 cells. Two days after transduction, deletion or insertion of $1 \mathrm{bp}$ were the two most frequent indel types in cells (Fig. 1e, S11). Following increased editing time (Dox-free groups, Fig. S10) and SpCas9 expression (Dox-induced groups, Fig. 1e), the frequency of other indel types rose significantly and $1 \mathrm{bp}$ insertion was the most dominant indel type which is in agreement with previous findings (Fig. 1e, S12) [37]. With the indel outcome, we were capable of analyzing the mutation consequence of all indels on protein translation. More than $70 \%$ of the total indels led to out-of-frame genotypes (Fig. S13). In conclusion, we demonstrated that TRAP-seq is a simple method for massively parallel quantification of gRNA editing outcomes in cells.

\section{Characterization of nucleotide features affecting SpCas9 efficiency and indel outcomes}

Development of more accurate rules for in silico CRISPR design relies heavily on datasets of gRNA activity and indel from a large number of gRNAs. The rich gRNA activity and indels profile data generated by TRAP-seq above were valuable for further improving the performance of CRISPR 
bioRxiv preprint doi: https://doi.org/10.1101/2020.05.20.103614; this version posted May $21,2020$. The copyright holder for this preprint (which was not certified by peer review) is the author/funder, who has granted bioRxiv a license to display the preprint in perpetuity. It is made available under aCC-BY-NC-ND 4.0 International license.

\section{Manuscript}

Online article

Confidential

design. We sought to investigate if the gRNA activity and indel outcomes measured by the TRAPseq can mirror previous findings about the effects of nucleotide features on CRISPR activity. Nucleotide features such as secondary structure [24] and GC content [40] of the guide sequences affect CRISPR editing efficiency. We analyzed the correlation between gRNA activity and GC content and secondary structure (deltaG energy) in the gRNA spacer. Our TRAP-seq results further confirmed that the gRNA spacer GC content (Fig. 2a, S14) and secondary structure (Fig. 2b, S15) affected SpCas9 gene editing efficiency in cells. The optimal GC content and deltaG energy is [5070\%] GC and [-5; -1] KJ/mol, respectively. Consistent with the previous finding [41], our TRAP-seq results revealed that the SpCas9 disfavors motifs of "TT" and "GCC" at the N17-N20 region (Fig. 2c, S16). Recent reports have discovered that indel profiles for a given gRNA is predictable [27, 28]. The SpCas9 predominantly generates blunt-end double-stranded DNA breaks (DSB) between the N17 and N18 nucleotide preceding the protospacer adjacent motif (PAM), which are most frequently repaired by the NHEJ and MMEJ pathways in mammalian cells [42]. We compared the indel profiles of approximately 12,000 gRNAs revealed by TRAP-seq to the predicted indel profiles by inDelphi, a machine learning program for SpCas9 indel prediction [27]. Our results show that the overall indel profiles (in the Dox-free or Dox-induced cells at day 8 and 10) are highly correlated with the predicted ones by inDelphi (median $\mathbf{r}=\mathbf{0 . 5 1 - 0 . 6 5}$, Fig. 2d and Fig. S17). Of note, the overall correlation between the TRAP indels in transduced cell at day 2 and indel profiles predicted by inDelphi was much lower (median $\mathbf{r}=\mathbf{0 . 3 1}$, Fig. S17), suggesting that the indel outcome also depends on the experimental conditions (e.g. Cas9 expression level, editing time etc.).

Among all indels, the 1bp insertion between N17 and N18 was the most abundant type (Fig. 1e). Previous studies had discovered that $1 b p$ insertion is not random [27]. We therefor asked whether the nucleotides of $1 \mathrm{bp}$ insertion among our 12,000 TRAP sites followed the same principle. First, we quantified the frequency of inserted adenine (A), thymine (T), cytosine $(\mathrm{C})$, guanine $(\mathrm{G})$ among all $1 \mathrm{bp}$ insertions. The results show that the $1 \mathrm{bp}$ insertion favor $\mathrm{T}$ and disfavor $\mathrm{G}$ (Fig. 2e). For a given TRAP site, however, there is a strong preference of one nucleotide type (also seen the CRISPR Atlas resource). Next, we divided all 12,000 TRAP sites into four groups based on the N17 or N18 nucleotide and quantified the frequency of inserted bases among all $1 \mathrm{bp}$ insertions. Our results show that the N17 nucleotide strongly defines the inserted base (Fig. 2f, S18), but less extensively affected by the N18 nucleotide (Fig. S19). Lastly, we divided all the 12,000 TRAP sites into 16 groups based on N17N18 sequence motifs and compared the indel frequency of $1 \mathrm{bp}$ insertion versus deletions. 
bioRxiv preprint doi: https://doi.org/10.1101/2020.05.20.103614; this version posted May 21, 2020. The copyright holder for this preprint (which was not certified by peer review) is the author/funder, who has granted bioRxiv a license to display the preprint in perpetuity. It is made available under aCC-BY-NC-ND 4.0 International license.

\section{Manuscript}

Online article

Confidential

Despite a constantly higher frequency of deletions over insertions, motifs of GN (N = A, T, C, or G) and $\mathrm{MC}(\mathrm{M}=\mathrm{A}$ or $\mathrm{C})$ at $\mathrm{N} 17 \mathrm{~N} 18$ favor deletions over 1bp insertion, as compared to $\mathrm{TN}$ and $\mathrm{MG}$ motifs (Fig. 2g, S20). Taken together, we show that high throughput TRAP-seq enables the identification and validation of features affecting SpCas9 editing efficiency and indel outcomes. The results corroborate previous findings that $\mathrm{SpCas} 9$ editing outcomes are predictable in cells.

a

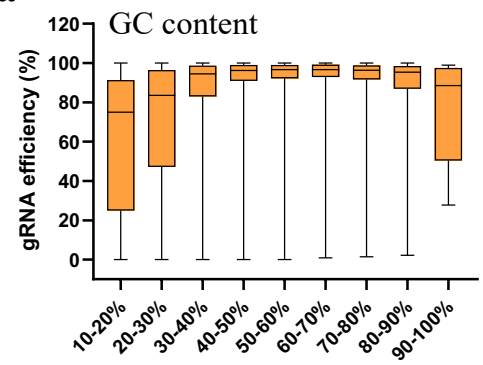

d

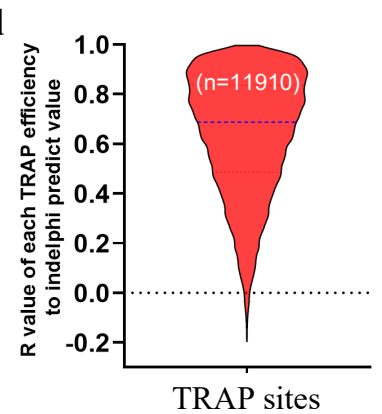

g

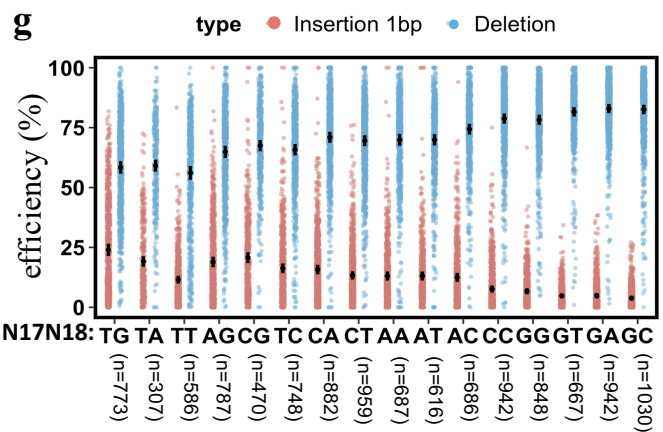

i

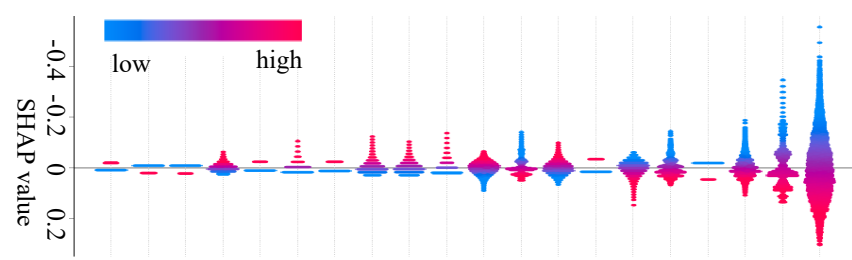

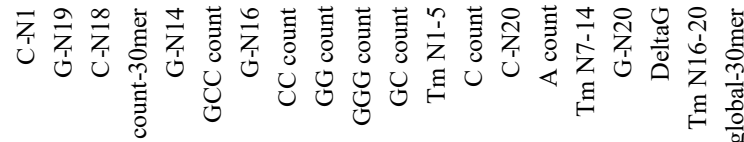

e

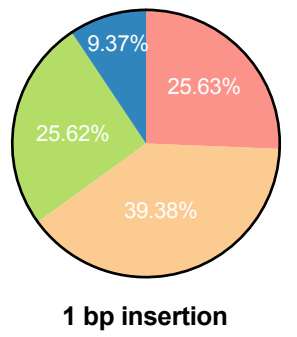

h

Trap-seq(Hek293)

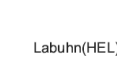

Doench V2(A375)

Hart(Hela2)

Chari (HEK293T)

Hart(Het116)

azimu b

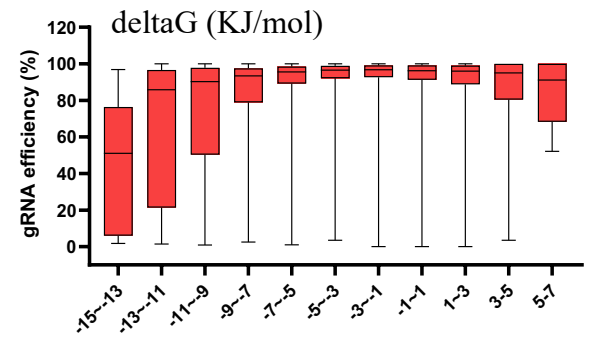

f

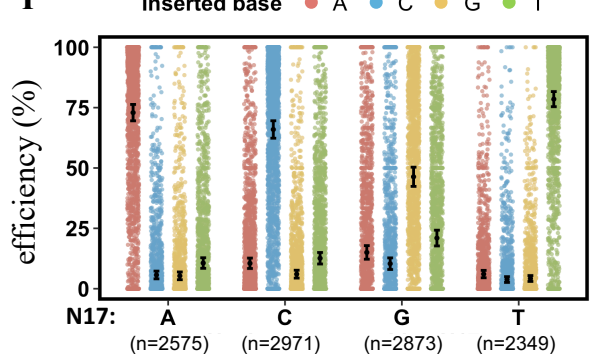

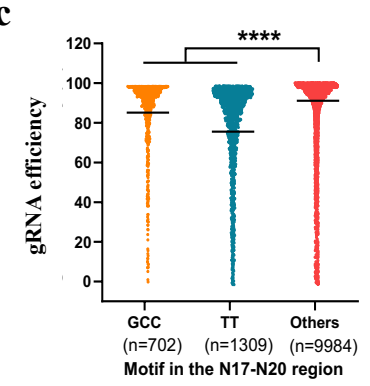


bioRxiv preprint doi: https://doi.org/10.1101/2020.05.20.103614; this version posted May $21,2020$. The copyright holder for this preprint (which was not certified by peer review) is the author/funder, who has granted bioRxiv a license to display the preprint in perpetuity. It is made available under aCC-BY-NC-ND 4.0 International license.

\section{Manuscript}

Online article

Confidential

\section{Figure 2 Characterization of features affecting SpCas9 efficiency and indel outcomes in cells}

a. Box plot between GC content (with an interval of 10\%) and SpCas9 gRNA efficiency measured in Dox-induced cells from Day 10. Results for other groups are shown in Fig. S14.

b. Box plot between deltaG energy (with an interval of 2) and SpCas9 gRNA efficiency measured in Dox-induced cells from Day 10. Results for other groups are shown in Fig. S15.

c. Comparison of SpCas9 efficiency between gRNAs harboring the GCC or TT motif in N17-N20 seed region and gRNAs without these two motifs. Data are shown for Dox-induced cells from Day 10. Results for other groups are shown in Fig. S16. “****”, p value less than 0.0001 .

d. Correlation between TRAP-seq indels and indels predicted by inDelphi from 11,910 sites. Data are shown for Doxinduced cells from Day 10. Results for other groups are shown in Fig. S17.

e. Pie chart of the proportion of $1 \mathrm{bp}$ insertion among four bases (A, T, C, G)

f. Correlation between the inserted 1 base and the nucleotide at N17 position. Data are shown for Dox-induced cells from Day 10. Results for other groups are shown in Fig. S18.

g. Effects of N17N18 dinucleotide motifs on the indel frequency of $1 \mathrm{bp}$ insertion and deletions (1-30 bp). The gRNAs are divided into 16 groups based on the N17N18 motifs. For each gRNA, the total indel frequencies of 1-30bp deletions and $1 \mathrm{bp}$ insertion were analyzed. " $\mathrm{n}$ " indicates the number of gRNAs included for each group.

h. Comparison of SpCas9 gRNA efficacy predictions in a regression schema for various datasets and prediction models. i. Top 20 features that weighted the most for the GNL machine learning model. Results are shown as the SHAP (SHapley Additive exPlanations) values. The 30mere comprises 4bp upstream, 20bp protospacer, 3 bp PAM, and 3 bp downstream sequences. Machine learning was based on gRNA efficiency data from Dox-induced cells at Day 10.

\section{An improved machine learning model to predict SpCas9 efficiency}

To further streamline the prediction of SpCas9 efficiency and the identification of nucleotide features important for gRNA activity, we randomly selected $80 \%$ of the 12K TRAP-seq gRNA efficiency and trained the GNL-Scorer [43] - a machine learning algorism that we previously developed based on the Bayesian Ridge regression (BRR) model and 2485 features. Our results showed that the GNLscorer trained with the TRAP-seq dataset (GNL-Scorer (Trap)) gave an accuracy prediction score of over $70 \%$ (Fig. S21). To benchmark the performance of the TRAP-seq dataset and the GNL prediction algorithm, we compared our dataset and GNL-Scorer (Trap) with seven previously published datasets (from HEL, NB4, TF1, MOLMB, A375, Hela, HEK293T, HCT116 cells) and five prediction tools (DeepCas9, Azimuth-2.0, TUSCAN, CRISPRater, SSC). Our results showed that the GNL-Scorer (Trap) achieved the best accuracy score in five datasets (second best for the remaining 3 datasets) and have the best generalized prediction outcome across all test datasets (Fig. 2h). Using the SHapley Additive exPlanations (SHAP) algorithm for explaining the feature output, our results further revealed features (such as melting temperature, $\mathrm{GC}$ content delta $\mathrm{G}$ energy, sequences motifs 
bioRxiv preprint doi: https://doi.org/10.1101/2020.05.20.103614; this version posted May $21,2020$. The copyright holder for this preprint (which was not certified by peer review) is the author/funder, who has granted bioRxiv a license to display the preprint in perpetuity. It is made available under aCC-BY-NC-ND 4.0 International license.

\section{Manuscript}

Online article

Confidential

etc.) that are important for the performance of our prediction model and SpCas9 efficiency (Fig. 2i). Our results taken together suggest the TRAP-seq dataset based GNL-Scorer performs generally well for gRNA knockout efficiency prediction that the 12K SpCas9 gRNA efficiency dataset revealed by TRAP-seq enable better understanding of features affecting gRNA efficiency in cells, improve the design of gRNAs of high knockout efficiency. This improved GNL-scorer algorithm for predicting SpCas9 efficiency has been deposited to and available at public domain GitHub.

\section{Quantification of CRISPR-mediated adenine base editing at 12,000 sites by TRAP-seq}

Unlike SpCas9 gene editing, we still lack large-scale data of CRISPR adenine base editing (ABE) efficiency. Such valuable data would enable us to develop better in silico ABE gRNA design tools. Since the TRAP site could confidentially recapitulate the ABE editing outcome of the corresponding endogenous site (Fig. S2), we sought to investigate the ABE editing outcomes in all 12,000 TRAP sites using the 12K TRAP-seq library. Although all the 12,000 gRNAs were not specifically designed for ABE editing, we reasoned that this "randomly" selected gRNA library would enable us to unbiasedly identify rules affecting ABE editing efficiency. To do this, we firstly transduced HEK293T-ABE cells with the 12K TRAP-seq lentivirus (MOI=0.3), and performed targeted amplicon sequencing of the TRAP DNAs from cells at 2, 7, and 11 days after transduction and cultured in Dox-free or Dox-addition medium (Fig. S22). For additional controls, we also performed similar experiments with wild-type HEK293T cells, with constant transduction coverage (4,690 cells per TRAP) and sequencing depth (approximately 1,000 reads per TRAP) (Fig. S23). 
$\mathbf{a}$

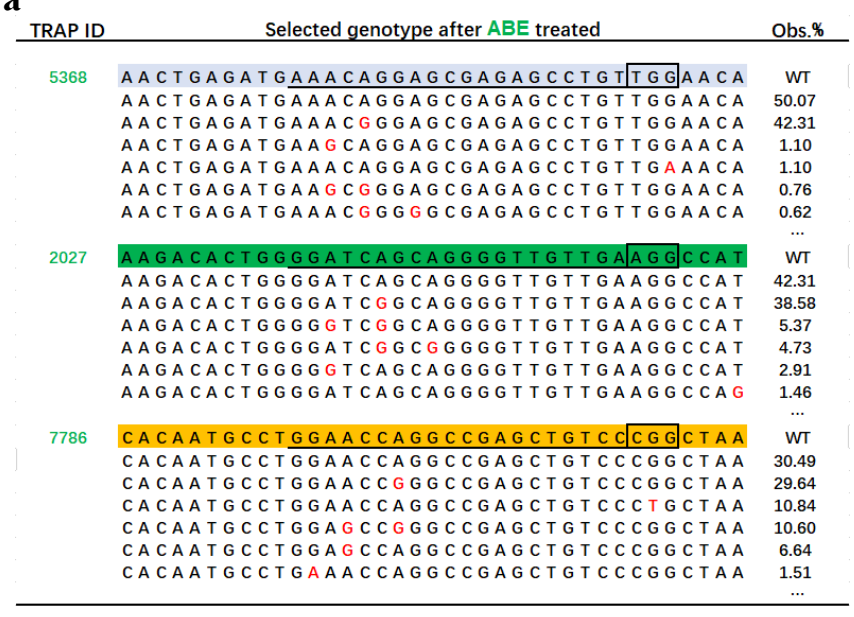

c
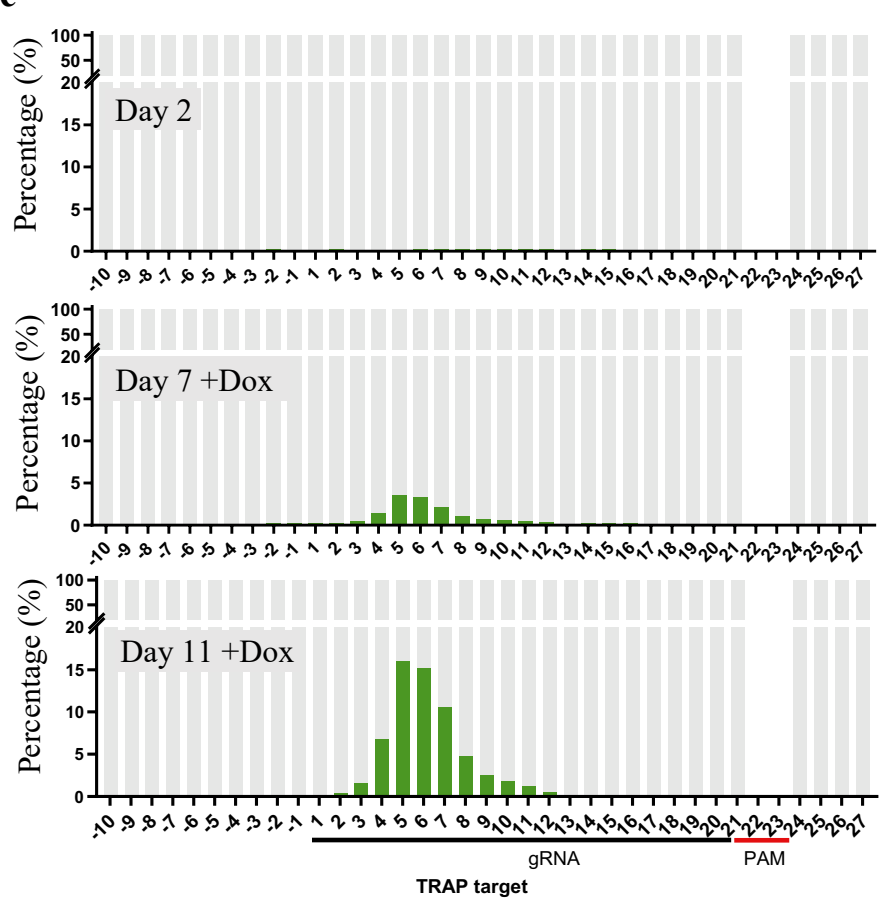

- $\mathrm{A} 2 \mathrm{G}=\mathrm{A} 2 \mathrm{C}=\mathrm{A} 2 \mathrm{~T}=\mathrm{A} 2 \mathrm{~A}$ b
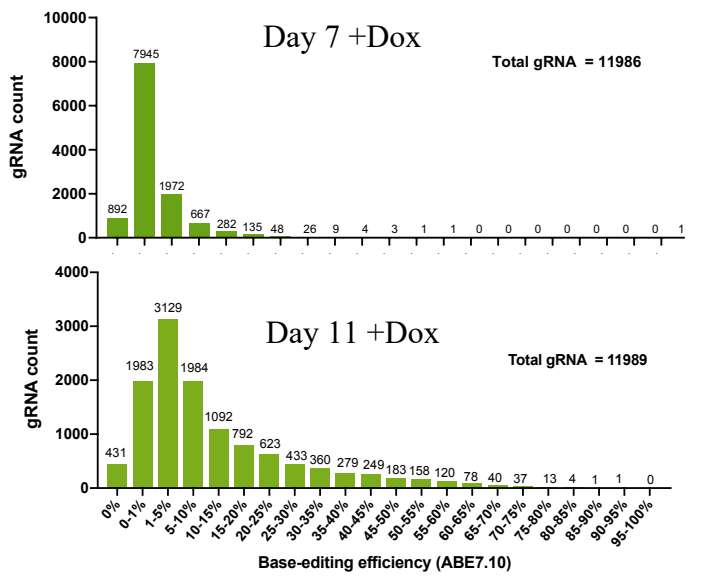

d
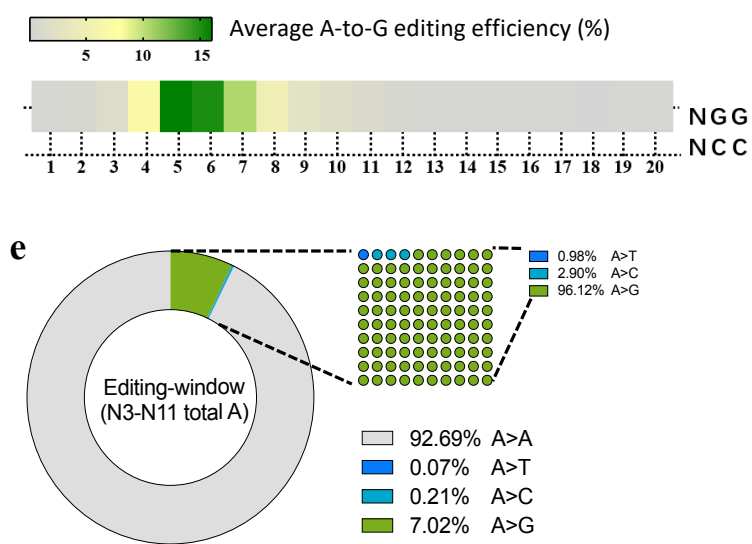

\section{Figure 3 Quantification of ABE efficiency at 12,000 sites by TRAP-seq}

a. Representation of top 5 adenine editing outcomes for three TRAP sites. Full ABE results for all 12,000 sites can be found at the CRISPR atlas resource.

b. Quantification of overall ABE efficiency for all gRNAs from Dox-induced HEK293T-ABE cells 7- and 11-days post transduction. Other groups are presented in Fig. S23.

c. Quantification of overall percentage of A-to-G, A-to-T, A-to-C, and A-to-A (unedited) events across the 37bp region of all TRAP sites. Results are from Dox-induced HEK293T-ABE cells at 2, 7- and 11-days post transduction. 
313 Next, we quantified the edited adenine events for each $37 \mathrm{bp}$ TRAP site in the 12K TRAP-seq library 314 (Fig. 3a, Table S3). Our results showed that substantial editing events (within an editing window 315 from N3 to N11) appeared 7 days after transduction in the HEK293T-ABE with Dox induction. The 316 editing efficiency increased 4-5 folds when extending the cultivation time to 11 days (Fig. 3b, S24). 317 Most importantly, the ABE editing window remained constant between N3 and N11 (Fig. 3c, S25), 318 supporting the notion that the $\mathrm{ABE}$ base editor is highly conserved with respect to its editing region 319 [19]. Our TRAP-seq results also validated that the highest ABE editing efficiency was observed for 320 adenines located at N5, N6 and N7 (Fig. 3d). Lastly, quantification of all 42,790 edited adenine sites 321 revealed that the $\mathrm{ABE}$ base editor conservatively generated A-to-G substitution (96.12\%), and a small 322 proportion of A-to-C (2.9\%) and A-to-T (0.98\%) substitutions (Fig. 3e). Although additional 323 experiments will be required to test the TRAP-seq library in more cell lines, these initial studies 324 suggest that the TRAP-seq is a highly valuable method for massively parallel quantification of 325 CRISPR adenine base editing efficiency in cells.

\section{Characterization of nucleotide features affecting ABE efficiency}

328 We also sought to characterize features that affect ABE efficiency. To enable comparisons, we first 329 selected two groups of gRNAs based on ABE efficiency: (1) high efficiency ABE gRNAs ( $\mathrm{n}=2,331$, 330 at least one edited adenine site had an efficiency over $20 \%$ with the protospacer region N1-N20) and 331 (2) low efficiency ABE gRNAs ( $n=2,589$, the efficiency of any edited adenine site within the 332 protospacer N1-N20 lower than 1\%). Next, we compared the base percentage between the low and 333 high efficiency gRNAs across the 37bp TRAP region for the low and high efficiency ABE gRNAs. 334 Not surprisingly, high efficiency gRNAs showed overrepresentation of Adenine within the editing 335 window N3-N7 (Fig. S26). Interestingly, high efficiency gRNAs favored Guanine over Thymine in 336 the seed region (N17 to N20), the distal protospacer region (N-1 to N4) and the N21 base of the PAM. 337 The presence of Cytosine in N20 was disfavored for high efficiency gRNAs. 

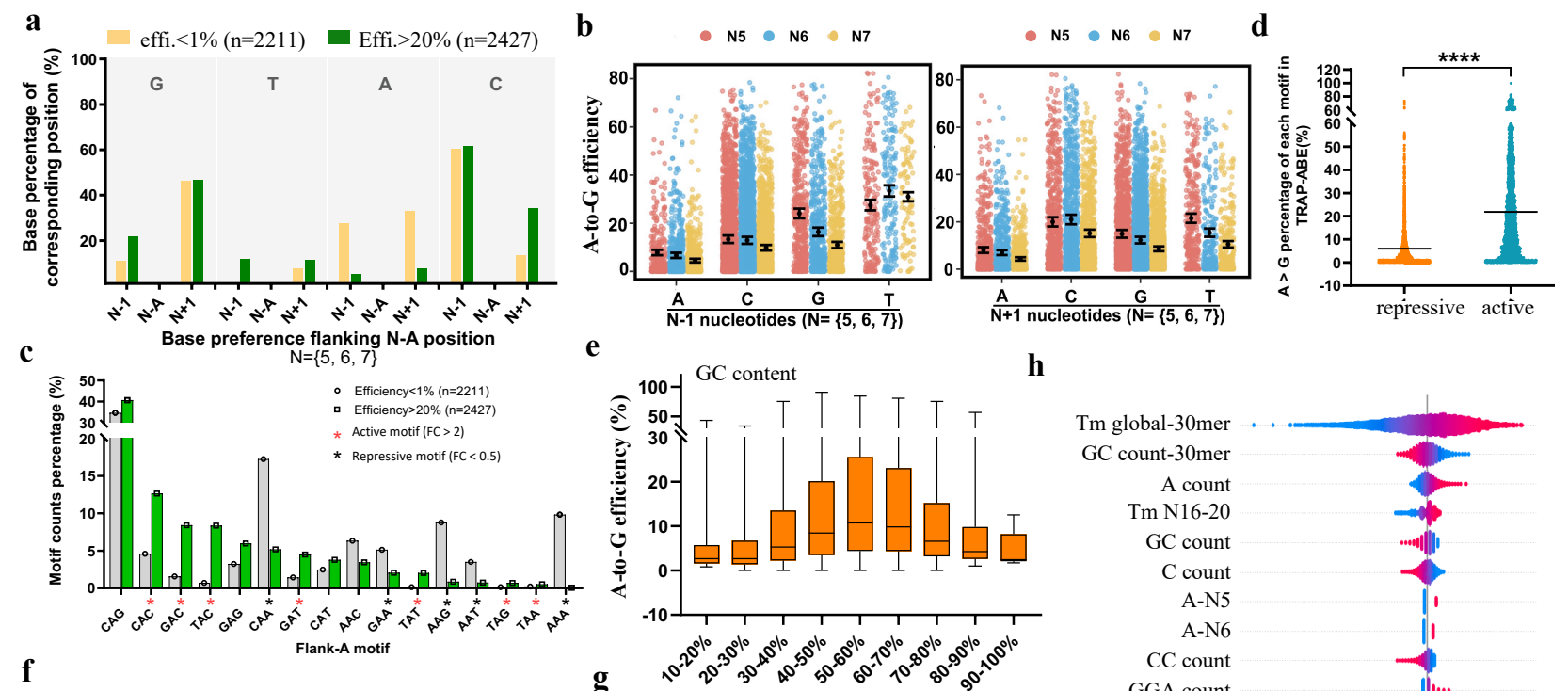

h
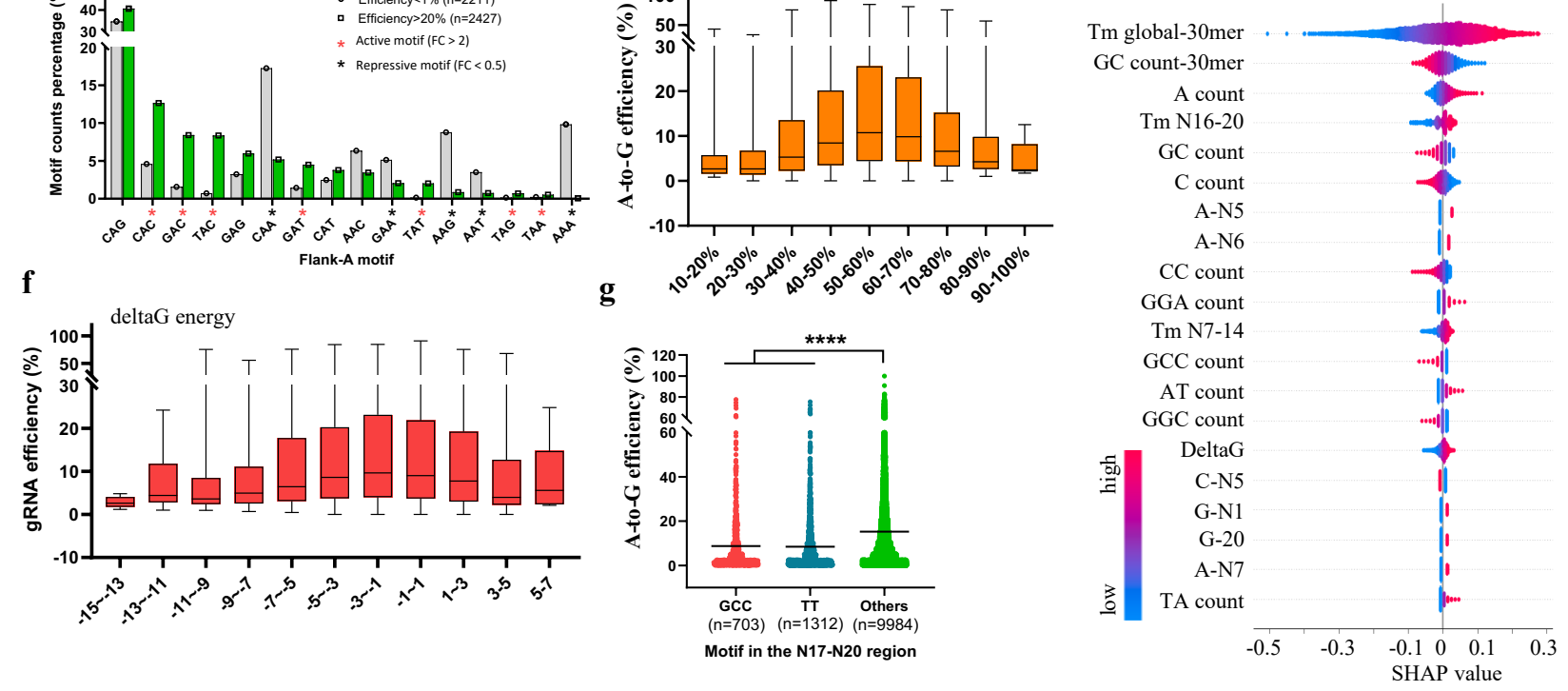

Figure 4 Characterization of nucleotide features affecting ABE efficiency

a. Proportion of frank-A (A bases located at the core editing window N5-N7) bases (A, T, C, G) between high (edited A efficiency $>20 \%$ for at least one A base within N5-N7) and low (edited A efficiency $<1 \%$ for any A base within N5N7) efficiency ABE gRNAs. Results are based on the data from the Dox-induced HEK293T-ABE cells from day 11. b. A-to-G editing efficiency for A bases located at N5-N7, grouped based on the flanking bases. Results are based on the data from the Dox-induced HEK293T-ABE cells from day 11.

c. Comparison of the frequency of NAN $(\mathrm{N}=\mathrm{A}, \mathrm{T}, \mathrm{C}, \mathrm{G})$ trinucleotide motifs between high and low efficiency ABE gRNAs (bold A referred to the deaminated bases within the N5-N7 core editing window). Red and black asterisks indicate the active and repressive motifs based on a cutoff of two-fold difference.

d. Scatter plot of edited A efficiency between sites within the active and repressive motifs. The number of " $n$ " indicates number of sites. “****”, p value less than 0.0001 .

e. Box plot of overall A-to-G editing efficiency for all TRAP gRNAs according to the gRNA spacer GC content (with $10 \%$ interval). Results are based on the data from the Dox-induced HEK293T-ABE cells from day 11. Complementing results for other groups can be found in Fig. S26.

f. Box plot of overall A-to-G editing efficiency for all TRAP gRNAs according to the gRNA spacer deltaG energy (with an interval of 2). Results are based on the data from the Dox-induced HEK293T-ABE cells from day 11. Complementing results for other groups can be found in Fig. S27. 
data from the Dox-induced HEK293T-ABE cells from day 11. Complementing results for other groups can be found in Fig. S28. “****”, p value less than 0.0001 .

h. Top 20 features that weighted the most based on the GNL machine learning model that affect the overall ABE efficiency in cells. Results are shown as the SHAP (SHapley Additive exPlanations) values. The 30mere comprises 4bp upstream, 20bp protospacer, 3 bp PAM, and 3 bp downstream sequences. Complementary SHAP results for each edited Adenine site within the N3-N11 window were shown in Fig. S30. Results are based on the ABE editing data from the Dox-induced HEK293T-ABE cells from day 11.

We next sought to investigate the effect of intrinsic nucleotide preference on base editing efficiency. To identify the intrinsic nucleotide preference for ABE7.10, we focused on the ABE efficiency at N5, N6 and N7, which were the three highest ABE sites (Fig. 3d). Using a similar strategy to enable comparison, we first selected two groups of gRNAs: N5-N7 high efficiency $(n=2427$, at least one edited adenine site had an efficiency over $20 \%$ within the core editing window N5-N7) and N5-N7 low efficiency $(n=2211$, the efficiency of any edited adenine site within core editing window N5N7 was lower than 1\%). Next, we analyzed the preference of flanking bases (A, T, C, G) at N5-N7 for the low and high efficiency gRNAs. Our results revealed that the high efficiency ABE gRNAs favored upstream keto $(\mathrm{K})$ bases $(\mathrm{G}, \mathrm{T})$ and downstream pyrimidine $(\mathrm{Y})$ bases $(\mathrm{T}, \mathrm{C})$ (Fig. 4a). The presence of flanking adenine was strikingly overrepresented in the low efficiency ABE gRNAs (Fig. 4a). To validate this observation, we analyze the correlation between A-to-G editing efficiency of all 12,000 TRAP sites in N5-N7 and their flanking bases. Consistent with the previous finding, the average $\mathrm{ABE}$ editing efficiency is higher if the edited adenine is flanked by Keto bases upstream and pyrimidine bases downstream (Fig. 4b), as compared to edited sites flanked by amino bases (A, C) upstream and purine bases $(\mathrm{A}, \mathrm{G})$ downstream. The overall ABE efficiency is much lower (approx. 2 to 7 folds) if the flanked base is adenine. Based on these observations, we further compared the frequency of tri-nucleotide flank-A motifs between the low and high ABE efficiency gRNAs. Using a cutoff of two folds, we categorized seven (BAC, KAT, TAR) and five (AAD, SAA) motifs (bold A refers to the deaminated adenine) as active and repressive flank-A motifs, respectively (Fig. 4c). To further validate that, we assessed A-to-G editing efficiency between all active or repressive flank-A motifs within our $12 \mathrm{~K}$ TRAP sites. Our results showed that the A-to-G editing efficiency is significantly higher $(\mathrm{p}<0.0001$, fold change $=4)$ in the active motifs as compared to the repressive ones (Fig. 4d, S27). 
Since the ABE editor shares principles of gRNA-guided DNA binding with SpCas9 nickase, we reasoned that many of the features (such as GC content, gRNA secondary structure (deltaG energy), N17-N20 motifs) that were known to influence SpCas9 editing efficiency should also affect ABE efficiency. To address that, we performed Pearson correlation analysis between the ABE efficiency and GC content and the deltaG energy of the guide sequence. Our results demonstrated that both GC content (Fig. 4e, S28) and secondary structure affect ABE efficiency (Fig. 4f, S29). We also investigated and validated that the presence of TT and GCC motifs at the N17-N20 seed region negatively affects ABE efficiency (Fig. 4e, S30).

\section{An improved machine learning model to predict ABE efficiency}

We sought to apply our GNL-scorer machine learning model [43] to systematically identify features of importance for ABE efficiency (GNL-scorer_ABE) and ABE efficiency prediction rules, as well as developed a new machine learning-based tool for predicting ABE efficiency. We randomly selected $80 \%$ (the remaining $20 \%$ used for model evaluation) of the ABE efficiency data and trained the Bayesian Ridge regression (BRR)-based GNL-scorer model with 2485 features (Fig. S31a). The ABE efficiency prediction was performed for both A-to-G edited site efficiency in N3-N11 and the overall ABE efficiency. Our results showed that the accuracy of predicting ABE is above $60 \%$ for the core ABE editing window (Fig. S31b), and the accuracy of predicting the over ABE efficiency is approximately 70\% (Fig. S21). The SHAP algorithm-based feature outputs further demonstrated that our machine learning results consistently revealed that features such as the global melting temperature, GC content, deltaG energy, and nucleotide compositions (such as the presence of Adenine in N5-N7, Guanine in N20) greatly affect the ABE efficiency (Fig. 4f and S32). Collectively, we demonstrate that the rich ABE editing efficiency data revealed by TRAP-seq enable us to systematically define factors influencing ABE efficiency and improve ABE gRNA design for future studies.

\section{Quantification of CBE-mediated recoding efficiency at 11,979 sites by TRAP-seq}

After demonstrating that the TRAP-seq method is versatile for massively parallel quantification of SpCas9 and ABE efficiency, we sought to test the performance of TRAP-seq for high throughput quantification of CBE efficiency. As mentioned earlier, all the gRNA spacers of our 12K TRAP-seq library were retrieved from the iSTOP database. This allows us to address the STOP recoding efficiency of all 11,979 gRNA and 3,832 genes by CBE in cells (Fig. 5a). First, based the optimized and constant conditions of lentivirus library transduction, we performed 10 parallel TRAP-seq-library 
422

423

424

425

426

427

428

429

430

431

432

433

434

435

436

437

438

439

440

441

442

443

444

445

446

447

448

based CRISPR CBE editing experiments in the Dox-inducible HEK293T-CBE cells (Fig. S33). Constant sequencing depths $(1,000 \mathrm{X}$ coverage $)$ and TRAP representation $(\mathbf{r}=\mathbf{0 . 9 6 - 0 . 9 7})$ were achieved for Dox-free and Dox-addition HEK293T-CBE cells 2 and 11 days after transduction by targeted amplicon sequencing of the TRAP region (Fig. S34). Next, to assess CBE editing, we quantified the efficiency of C-to-T edit, as well as C-to-G and C-to-A edits, of all $\mathrm{C}$ bases within the 37bp of all 11,979 TRAP sites (Fig. 5b, Table S4, full results were shown in the CRISPR Atlas database). As seen from the overall (Fig. 5c, S35) and C-to-T site (Fig. 5d) CBE efficiency plots, quantification of the average efficiency of all edited Cs for the 11979 gRNAs revealed that there was an even distribution of gRNAs editing efficiency from 5\% to $75 \%$. There were 371 gRNAs with very low CBE efficiency (0-5\%) (Fig. 5c). The C-to-T CBE efficiency was primarily detected in the Doxinduced HEK293T-CBE cells at 11 days after transduction, indicating there was very minor leakiness of CBE editor expression (Fig. 5c, S35). Of particular note, compared to ABE, the editing window of CBE was broader (N1 to N16) but the highest cytosine editing efficiency was similarly found at N6 (Fig. 5d, S36a). Quantification of all edited Cs revealed that the majority (93.18\%) were C-to-T edits, however, low frequency of unspecific C-to-G (4.54\%) and C-to-A (2.27\%) edits were also observed for the CBE (BE4-gam) base editor (Fig. S36b).

We next investigated the stop-codon recoding efficiency by CBE in cells. With the current setups in HEK293T-CBE cells, the median STOP efficiency of all 11,979 gRNA was approximately 22\% (Fig. 5e, S37, S38a). A total of 3,481 genes (90\%) were successfully knocked out by stop-codon recoding with CBE (Fig. 38b). We also sought to analyze the efficiency of the 6 types of recoding into stop codons (Fig. 5a). As seen in Fig. 5f, the recoding efficiencies of CCA-to-CTA, CCA-to-TCA and CCA-to-TTA were significantly higher (p value $<\mathbf{0 . 0 0 0 1}$ ) than CGA-to-TGA, CAG-to-TAG and CAA-to-TAA (bold $\mathbf{C}$ refers to the deaminated cytosines). Taken together, we here demonstrated that the TRAP-seq technology enables massively parallel quantification of CBE-mediated recoding capacity in cells. 
a

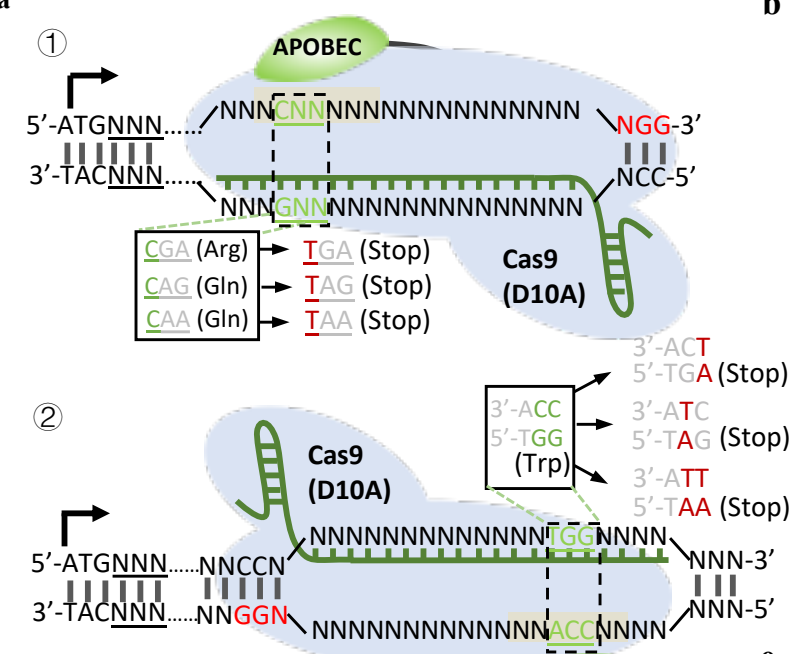

b

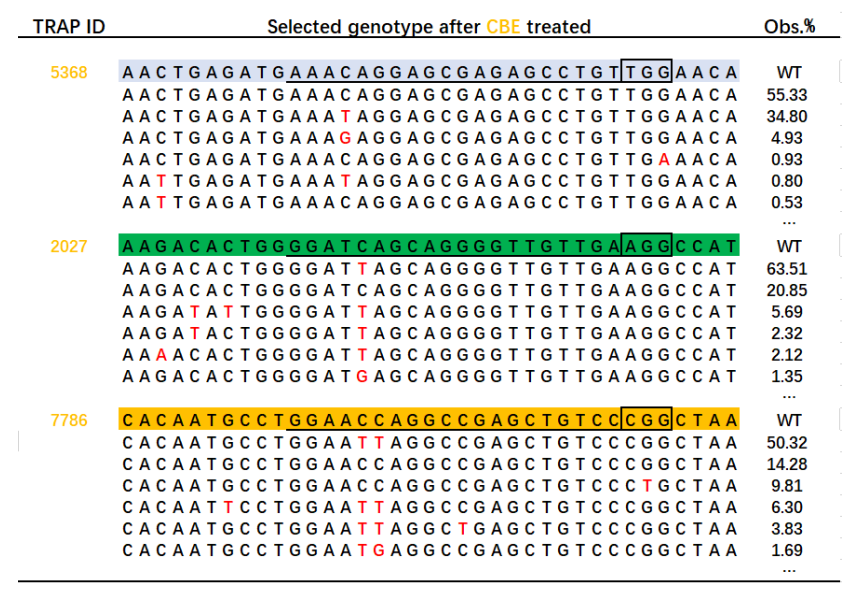

e TRAP ID Selected data of TRAP-CBE mediated STOP codon conversion (CGA>TGA) Obs.\% \begin{tabular}{l|c|c|c|c|c|c|c|cc} 
ABCB5 & Tyr & Arg & Asp & His & lle & Gly & Val & & \\
\hline A T G T G C G G C A T T A T C G A G A C C A T A T T & G G A G & T G G & T T A G & WT
\end{tabular} A T G T GCGGCATT ATCGAGACCATATTGGAGT GGT T A G WT A T GT GCGGCATT ATT GAGACCATATTGGAGT GGT T AG 46.40 $\begin{array}{llllll}\text { A T G T GCGGCA T T A T T G A G A T C A T A T T G G A G T G T T A G } & 1.99\end{array}$ \begin{tabular}{ll|l|l|l|l} 
A T G T G T G G C A T T A T T G A G A C C A T A T T G G A G T G G T T A G & $\mathbf{1 . 8 7}$
\end{tabular}

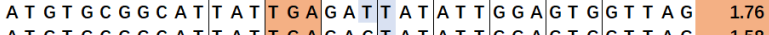

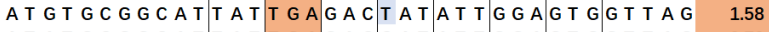
A T A T GCGGCATT A T T GAGAC CA TA T T G GAG T GGT TAG 0.70 A T G T G CGGCATT A T T GAGACCATATTGGAGT GGT TAA 0.53 A T G A G C G G C ATT A T T G A G A C C A T A T T G G A G T G G T T A G 0.53 A T GT GCG GCATT A T T G AGAGTATAT TGGAGTGGT T A G 0.12 A T G T G C G G C A T T A T C G A G A T T A T A T T G GAG T GG T T A G 0.12 A TGTC CGG ATTATT GAGACATATTG GAGTGGT AG 0.12 A T GT CCGGCATTATT ATT ATT AT GTGCGG ATGTGCGGCAGTATTGA ACGTGCGGCAAT AC T GAGAC GATAT T GGAGT G GT T AG 0.06

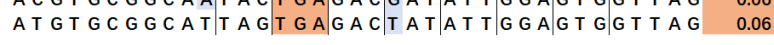

f
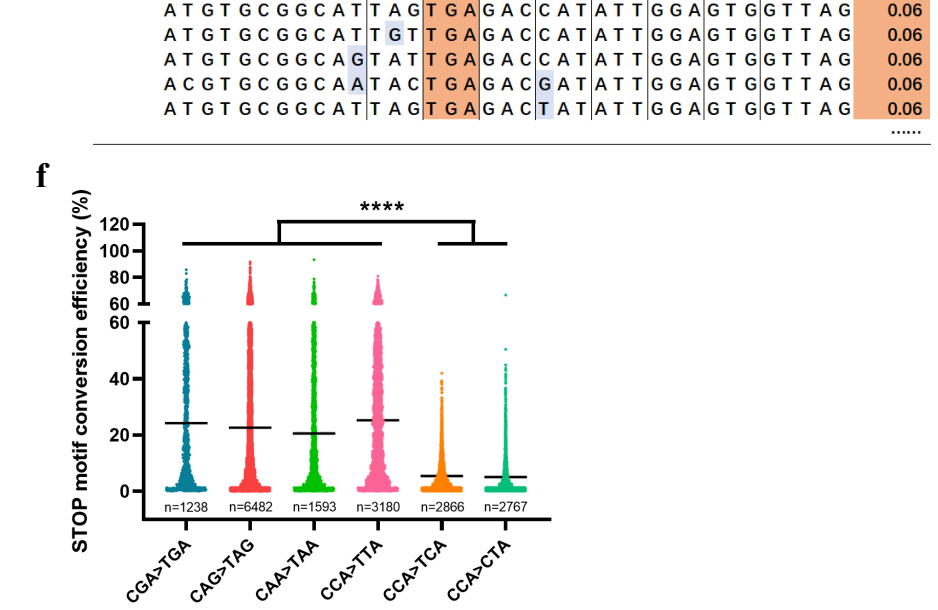
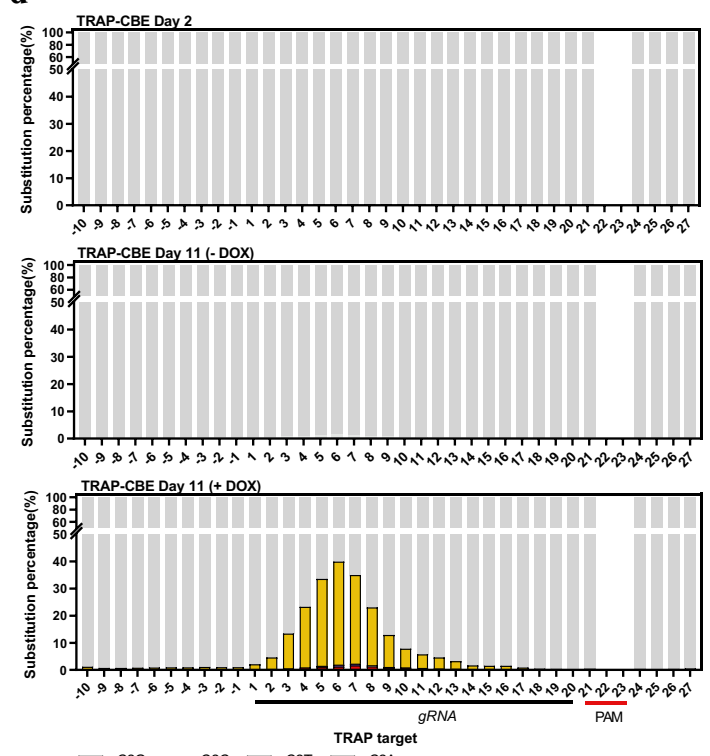

450 Figure 5 Quantification of CBE-mediated recoding efficiency at 12,000 sites by TRAP-seq

451 a. Schematic illustration of the 6 stop-codon recoding schemes by CBE. Cas9 (D10A) is the Cas9 nickase used in the 452 testing CBE editor. The stop-codon recoding scheme is draw based on sense and anti-sense strands. "ATG", translation 453 start site; "NGG", PAM of SpCas9.

454 b. Representation of top 5 cytosine base editing outcomes for three TRAP sites. Full CBE frequency results can be 455 found in the CRISPR Atlas database. 
c. Quantification of overall CBE efficiency for all gRNAs in Dox-induced HEK293T-CBE cells at 11 days. Other groups are presented in Fig. S33.

d. Quantification of overall percentage of C-to-T, C-to-G, C-to-A, and C-to-C (unedited) events across the 37bp region of all TRAP sites. Results are based on HEK293T-CBE cells from 2 days post transduction, and transduced HEK293TCBE cells from 11 days cultured in Dox-free or Dox-addition medium.

e. Representation of CBE-mediated recoding efficiency at one TRAP site measured by TRAP-seq. Complementary results referred to Fig. S35 and CRISPR atlas database.

f. Comparison of C-to-T efficiency between sites within the 6 stop-codon recoding types. " $n$ " indicates the number of sites within each group. “****”, p value less than 0.0001 .

\section{Characterization of nucleotide features affecting CBE efficiency}

The large-scale CBE efficiency data from 56,887 edited Cs and 11,979 gRNAs enable us to characterize the features that affect CBE efficiency in cells. To simplify the analysis and characterization, we first selected two opposing types of CBE gRNAs based on their efficiencies: low efficiency ( $\mathrm{n}=1,844$, the efficiency of any edited $\mathrm{C}$ within the protospacer is $<5 \%$ ) and high efficiency ( $n=1,731$, At least one edited $C$ efficiency within the protospacer is $>60 \%)$. Next, we compared the base preference across the $37 \mathrm{bp}$ TRAP regions between the two types of gRNAs. Our results clearly show that the high efficiency CBE gRNAs favor the presence of Cytosine (N4-N8), but disfavor Guanine (N3-N7) and Adenine (N5-N6) within the core editing window (Fig. S39). The presence of Thymine within the seed region (N8-N20) of protospacers was underrepresented in the high efficiency CBE gRNAs, which was in contrast to the N3-N6 region. Similar to our findings in ABE, high efficiency CBE gRNAs favor the presence of Guanine at proximal PAM region (N19N21) and disfavored Cytosine at N20.

Since the core editing window of CBE is N4-N8 (Fig. S36), we focused on the deaminated Cytosines within N4-N8 when further analyzing the effect of flanking bases on CBE efficiency. To enable comparison, we selected C-to-T edited sites of low $(<5 \%, \mathrm{~N}=4,898)$ and high $(>50 \%, \mathrm{~N}=5,058)$ efficiency within the N4-N8 region. Our results show that the presence of Thymine upstream is strikingly overrepresented in the highly efficient C-to-T editing, whereas the presence of Guanine upstream is only present in the low efficiency CBE sites (Fig. 6a). In addition, the highly efficient CBE sites are less frequently flanked by Adenine downstream and more frequently flanked by Cytosine, as compared to low efficiency CBE sites (Fig. 6a). To validate this observation, we calculated the C-to-T editing efficiency at N4-N8 for all 12,000 TRAP sites. Consistent with previous observations, the overall efficiency of CBE sites flanked by Thymine upstream is approximately two- 
fold higher than with other flanking bases. Of note, sites flanked by Guanine upstream (as well as downstream, but to less extent) show much lower CBE efficiency (fold changes $=2-12$ folds) (Fig. 6b). This provides highly valuable knowledge for designing CBE gRNAs with better editing outcome.

a

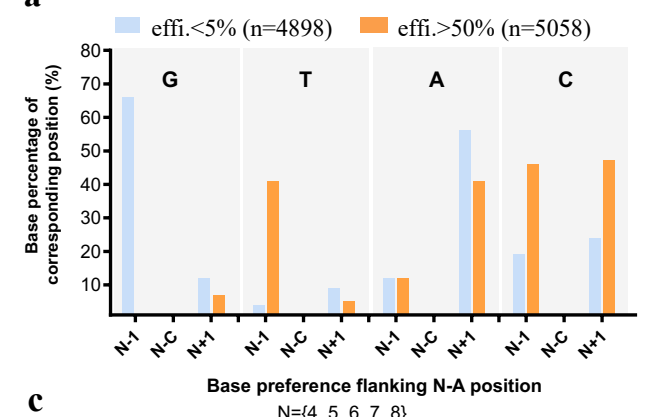

c

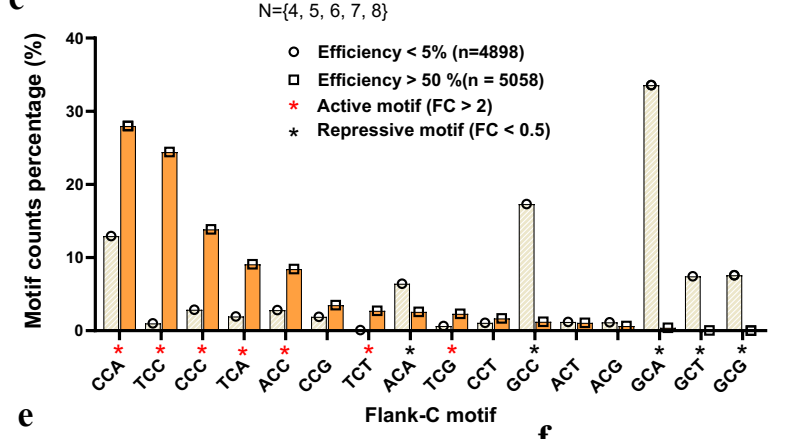

e

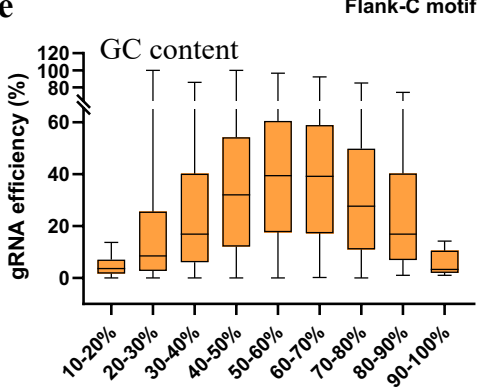

g

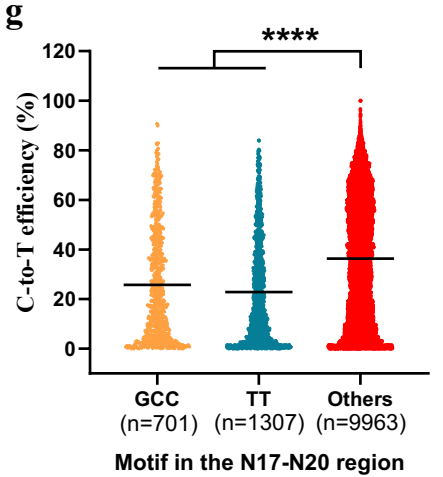

b

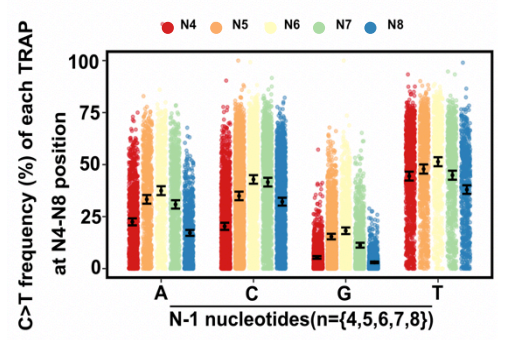

d

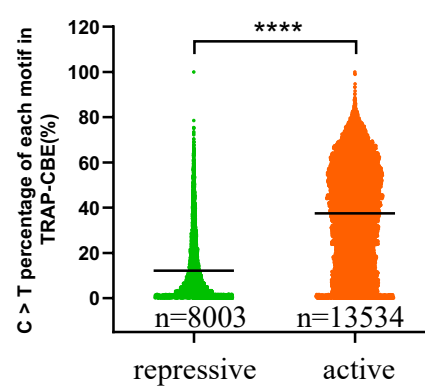

f $\quad{ }_{100} \operatorname{deltaG}(\mathrm{KJ} / \mathrm{mol})$

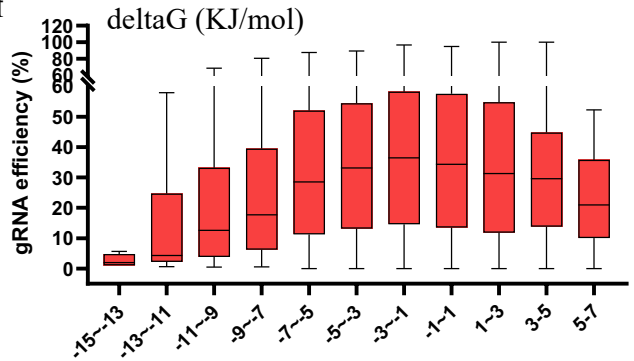

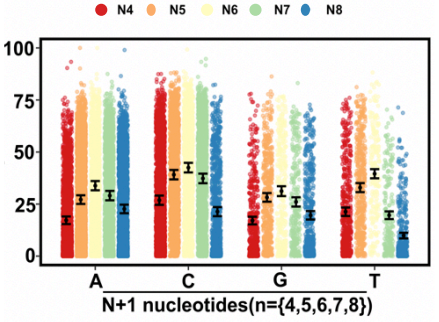

h

Tm global-30mer $\mathrm{GC}$ count

Tm N16-20

Tm N1-5

GC count-30mer C-N6 C-N5 DeltaG TC count Tm N7-14 G-N20 GG count GCC count Tm N1-20

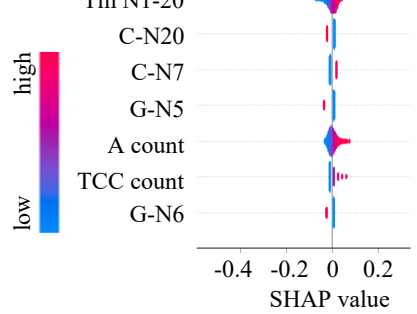

Figure 6 Characterization of nucleotide features affecting CBE efficiency in cells by TRAPseq

a. Proportion of frank-C (N4-N8) bases (A, T, C, G) between high (at least one edited C base within N4-N8 was $>50 \%$ edited) and low (edited $\mathrm{C}$ efficiency for any $\mathrm{C}$ base within N4-N8 was less than 1\%) efficiency CBE gRNAs. Results are based on the data from the Dox-induced HEK293T-CBE cells from day 11. 
b. C-to-T editing efficiency for C bases located at N4-N8, grouped based on the flanking bases. Results are based on the data from the Dox-induced HEK293T-CBE cells from day 11.

c. Comparison of the presence of the $16 \mathrm{NCN}$ trinucleotide motifs between high and low efficiency CBE gRNAs (bold $\mathrm{C}$ referred to the deaminated cytosine within the N4-N8 core editing window). Red and black asterisks indicate the active and repressive motifs based on a cutoff of two-fold difference.

d. Scatter plot of edited C efficiency between sites within the active and repressive motifs. The number of " $n$ " indicates number of sites. “****”, p value less than 0.0001 .

e. Box plot of overall C-to-T editing efficiency for all TRAP gRNAs according to the gRNA spacer GC content (with $10 \%$ interval). Results are based on the data from the Dox-induced HEK293T-CBE cells from day 11. Complementing results for other groups can be found in Fig. S36.

f. Box plot of overall C-to-T editing efficiency for all TRAP gRNAs according to the gRNA spacer deltaG energy (with an interval of 2). Results are based on the data from the Dox-induced HEK293T-CBE cells from day 11. Complementing results for other groups can be found in Fig. S38.

g. Scatter plot of overall CBE efficiency between gRNAs have the GCC or TT motifs within the seed region N17-20 and gRNAs without these two motifs. " $n$ " indicates the number of gRNAs within each group. Results were based on the data from the Dox-induced HEK293T-CBE cells from day 11. Complementing results for other groups can be found in Fig. S41.

h. Top 20 features that weighted the most based on the GNL machine learning model, which affect the overall CBE efficiency in cells. Results are shown as the SHAP (SHapley Additive exPlanations) values. The 30mere comprises $4 \mathrm{bp}$ upstream, 20bp protospacer, 3 bp PAM, and 3 bp downstream sequences. Complementary SHAP results for each edited cytosine site within the protospacer N1-N20 were shown in Fig. S42. Results are based on the CBE editing data from the Dox-induced HEK293T-CBE cells from day 11.

Since the flanking bases played an important role on CBE efficiency, we reasoned that there exists a preference of tri-nucleotide flank-C motifs $(\mathrm{NCN} ; \mathrm{N}=\mathrm{A}, \mathrm{T}, \mathrm{C}, \mathrm{G}$; bold $\mathrm{C}$ refers to the deaminated cytosine located at N4, N5, N6, N7 or N8) for active or repressive CBE. To identify these motifs, we compared the frequency of all $16 \mathrm{NCN}$ motifs between the low $(\mathrm{N}=4898$, efficiency $<5 \%)$ and high $(\mathrm{N}=5058$, efficiency $>50 \%)$ efficiency cytosine editing sites in N4-N8. Based on a two-fold difference, we identified seven (TCN, CCM and ACC; N=A, T, C, G; M = A, C) and five (ACA and GCN) as active and repressive motifs, respectively (Fig. 6c). To validate this, we further compared the C-to-T editing efficiency between the active and repressive motifs for all 21537 edited $\mathrm{C}$ sites in our 12K TRAP-seq library. The C-to-T editing efficiency of the active motifs were significantly higher (fold change $=3, \mathrm{p}<0.0001$ ) than those within the repressive motifs (Fig. 6d, S40). We also sought to investigate if the CBE efficiency shared features with SpCas9 editing efficiency using our CBE 12K TRAP-seq data. Thus, we analyzed the correlation between CBE efficiency and the GC content, the gRNA spacer secondary structure, as well as the proximal PAM motifs. Our results show 
535

536

537

538

539

540

541

542

543

544

545

546

547

548

549

550

551

552

553

554

555

556

557

558

559

560

561

562

563

564

565

566

that, similar to SpCas9 and ABE, the CBE efficiency is affected by the gRNA spacer GC content (Fig. 6e, S41) and secondary structure (Fig. 6f, S42). The CBE efficiency of gRNAs is significantly $(\mathbf{p}<\mathbf{0 . 0 0 0 1})$ lower with TT or GGC motifs at the proximal (17-N20) PAM region (Fig. 6g, S43).

\section{An improved machine learning model to predict CBE efficiency}

We further took advantage of our GNL-scorer machine learning model to development a prediction tool and systematically evaluate the effect of 2485 features on CBE efficiency. Based on randomly selecting $80 \%$ of $12 \mathrm{~K}$ gRNAs CBE efficiency for model training and $20 \%$ for model evaluation, our results showed that the accuracy prediction score of CBE efficiency by the GNL machine learning model reaches approximately $80 \%$ (Fig. S21). Apart from predicting the overall CBE efficiency, our machine learning-based prediction tool provides highly precise predicting outcome of the sitespecific CBE efficiency within the editing window (Fig. S44). Consistently, our machine learning results further showed that features such as melting temperatures, GC content, deltaG energy, nucleotide composition (e.g. the presence of cytosine at N5-N7, nucleotide counts, motifs) greatly affect CBE efficiency (Fig. 6h and Fig S45). Finally, the machine learning-based CBE efficiency prediction algorism (GNL-scorer_CBE) has also been deposited to the GitHub to facilitate the design of CBE gRNAs of high efficiency. Taken together, we hereby demonstrate that high-throughput quantification of CBE efficiency by TRAP-seq enables the better understanding and design of highly efficient CBE gRNAs in cells.

\section{The CRISPR Atlas}

As part of this work, a human CRISPR atlas database (http://www.crispratlas.com/crispr) has been launched to present and integrate all the SpCas9, ABE and CBE efficiency and editing outcomes for 12,000 gRNAs in HEK293T cells. This CRISPR atlas is presented with a gene-centered and gRNAcentered summary of the overall efficiency of gRNAs and editing outcomes. For SpCas9, total efficiency, indel (1-30bp deletions, 1-10bp insertions) profiles were presented for each gRNA. For $\mathrm{ABE}$ and $\mathrm{CBE}$, the overall gRNA efficiency and graphical presentation of base substitution efficiency across the 37bp TRAP region were shown for all 12,000 TRAP sites. We believe that the CRISPR atlas database generated by this study will complement the existing CRISPR resources in gRNA design [44], efficiency prediction [45, 46] and indel prediction [27], thus streamlining the application of CRISPR in functional studies. 
567

568

569

570

571

572

573

574

575

576

577

578

579

580

581

582

583

584

585

586

587

588

589

590

591

592

593

594

595

596

597

598

\section{DISCUSSION}

In conclusion, the work described here demonstrates the broad applicability of the TRAP-seq system for massively parallel quantification of SpCas9, $\mathrm{ABE}$ and $\mathrm{CBE}$ efficiency in human cells. Recent studies published by other groups have demonstrated that the surrogate target sites can well mimic the SpCas9 indel outcomes at the corresponding endogenous sites, and thus predict the SpCas9 indel profile for a given gRNA in cells $[26,28,40]$. Consistent with that, we demonstrate corroborating findings with our TRAP-seq method and further expand the data of SpCas9 knockout efficiency and indel profiles with 12,000 gRNAs. This will aid the improvement of CRISPR gRNA design for gene knockout purposes with machine learning models [40,45]. With such a large amount of SpCas9 efficiency data, it is possible to systematically identify both previously known as well as novel features that affect CRISPR gene editing efficiency. Importantly, according to our knowledge, this is the first time that both $\mathrm{ABE}$ and $\mathrm{CBE}$ efficiencies are measured at such a large scale in cells. Based on the $12 \mathrm{~K} \mathrm{ABE}$ and CBE TRAP-seq data, our analyses identify several novel features (such as the preference of flanking bases, active/repressive tri-nucleotide motifs) that strongly influence ABE and CBE efficiency in cells, respectively. We believe that incorporating the nucleotide features of importance for $\mathrm{ABE}$ and $\mathrm{CBE}$ efficiency from this study will improve the performance of in silico base editing designers such as BE-Designer [47] and Beditor [48]. However, we acknowledge that there might be a difference in the DNA repair machinery between different cell types and organisms, which will potentially affect the SpCas9, $\mathrm{ABE}$ and $\mathrm{CBE}$ efficiency and outcome. Additional experiments will be required to test the SpCas9, $\mathrm{ABE}$ and $\mathrm{CBE}$ efficiency with TRAP-seq in more cell lines in the future.

The concept of using surrogate target sites to capture the gene editing outcomes is highly attractive. We and other groups have generated dual-fluorescence-based surrogate systems for rapid evaluation of ZFNs, TALENs and CRISPR-Cas9 activity in cells [29, 49, 50]. The DSBs generated by CRISPRCas9 were predominantly repaired by the NHEJ and MMEJ pathways, which will lead to the introduction of small indels at the DBS site. However, large deletions or chromosomal rearrangements have also been reported in CRISPR editing as outcomes of repaired mediated by e.g. HDR or SSA in cells $[51,52]$. The TRAP-seq system developed in this study is based on a $37 \mathrm{bp}$ surrogate target site. Thus, SpCas9 editing outcomes such as large deletions or chromosomal arrangements will not be captured by our method. However, for ABE and CBE, the editing outcomes would not be affected by such a size-related problem. 
600 Earlier, we have discovered that chromatin accessibility at the editing sites affect CRISPR gene 601 editing efficiency [24]. Since the TRAP-seq library were randomly inserted in the genome of the 602 targeted cells, the chromatin accessibility state of the surrogate site might be different from the 603 endogenous target site. It would be interesting to apply the TRAP-seq system to systematically 604 analyze the epigenetic factors (e.g. DNA methylation, chromatic accessibility) on ABE, CBE 605 efficiency in future studies.

606

607 In this study, we demonstrate the TRAP-seq system with applications in massively parallel 608 quantification of editing efficiency for SpCas9 and base editors (ABE and CBE) derived from the 609 SpCas9. The current CRISPR-based gene editing toolbox has been greatly expanded with the 610 engineered SpCas9 (e.g. xCas9, eSpCas9, SpCas9-HF1), the SpCas9 orthologs (e.g. SaCas9, StCas9, 611 NmCas9) and other Cas proteins (e.g. Cas12a) [53-55]. However, features affecting the editing 612 efficiency and indel outcomes are still rarely explored for most of these Cas proteins, which will limit 613 the applications of this great toolbox. We believe that the TRAP-seq will become an important 614 technology for the whole CRISPR gene editing society to better understand how CRISPR gene editing 615 works in cells. The CRISPR atlas database generated by this study will become a CRISPR-centered 616 portal, in which we provide experimentally validated gRNAs for CRISPR gene editing. Taken 617 together, the TRAP-seq technology, the SpCas9/ABE/CBE efficiency of 12,000 gRNAs, and the 618 CRISPR atlas database will enable us to better functionally understand how CRISPR works in cells 619 and improve CRISPR in both research, therapeutic and drug discovery applications. 
622

623

624

625

626

627

628

629

630

631

632

633

634

635

636

637

638

639

640

641

642

643

644

645

646

647

648

649

650

651

652

\section{MATERIALs and METHODS}

\section{Vector construction}

The empty pLenti-TRAP-seq vector backbone (shown in Fig. S1) was generated by a serial of cloning. Briefly, we replaced the SpCas9 open reading frame (ORF) in pLentiCRISPRv2-puro (Addgene plasmid \# 98290) plasmid with an enhance green fluorescence protein (EGFP) ORF based on XbaI and $B m H I$ digestion and T4 ligation. Next, we replaced the gRNA cassette in the EGFP-inserted pLentiCRISPRv2-puro with a synthetic Golden-Gate Assembly cassette, and hence generated a lentivirus-based vector (hereafter referred as pLenti-TRAP-seq) allowing the insertion of TRAP DNA to the Golden-Gate cloning site by GGA. The full sequencing of the pLenti-TRAP-seq vector can be downloaded from our CRISPR atlas database (www.crispratlas.com/crispr). Original plasmid stock can be acquired from the corresponding authors' lab.

The doxycycline inducible SpCas9, ABE, and CBE vectors were generated by subcloning. Briefly, based on a PiggyBac transposon system (full GenBank vector sequences can be found at the CRISPR atlas website), which consists of an all-in-one expression system: (1) An expression cassette of a TRE promote-driven protein expression cassette with multiple cloning sites (MCS). (2) An expression cassette of a consecutive promoter-driven Tetracycline-Controlled Transcriptional Activation and hygromycin. The coding sequences of SpCas9 (Addgene plasmid ID \# 41815), ABE 7.10 (Addgene plasmid ID \# 102919), and CBE (Addgene plasmid ID \# 100806) were PCR amplified and inserted to the MCS of the PiggyBac transposon system. All vectors were validated by Sanger sequencing.

\section{TRAP 12K oligos design and microarray synthesis.}

A typical TRAP oligo consists of the BsmBI recognition site "cgtctc" with 4 bp specific nucleotides "acca" upstream, following the GGA cloning linker "aCACC", one bp "g" for initiating transcription, then the 20 bp gRNA sequences of "gN20", 82bp gRNA scaffold sequence, $37 \mathrm{bp}$ surrogate target sequences (10bp upstream sequences, 23 bp protospacer and PAM sequences, 4 bp downstream sequence), the downstream linker "GTTTg" and another BsmBI binding site and its downstream flanking sequences "acgg”. An example of the typical TRAP oligo sequence was shown below: “accacgtctcaCACCgGTCCCCTCCACCCCACAGTGGTTTTAGAGCTAGAAATAGCAAGTTA AAATAAGGCTAGTCCGTTATCAACTTGAAAAAGTGGCACCGAGTCGGTGCTTTTTTACT TTTATCTGTCCCCTCCACCCCACAGTGGGGCCACGTTTggagacgacgg”. Sequences in the 
653 654 655 656 657 658 659 660 661 662 663 664 665 666 667 668 669 670 671 672 673 674 675 676 677 678 679 680 681 682 683 684

black frame is the $20 \mathrm{bp}$ gRNA spacer. The underline sequence is the $37 \mathrm{bp}$ surrogate target site, which is termed as "TRAP target" shortly in this study.

For the 12K TRAP oligo design, we used bioinformatic tools to automatically generate the 12K TRAP oligo pools. Briefly, 1) we selected approximately 7,000 genes from the a drugable gene database (http://dgidb.org); 2) Discard all the exons which the DNA length was less than $23 \mathrm{bp}$ with filtering; 3) Select the first three coding exons of each gene. If the exons number is less than 3 , keep all the exons; 4) Extract all the possible gRNA sequences (including the PAM sequence "NGG") in these filtered exons sequence, analyzes and predictd the off-target sites of each gRNA using FlashFry version 1.80 (https://github.com/mckennalab/FlashFry), discarded gRNAs with potential off-target of 0-3 bp mismatches in human genome; 5) Rank each gRNA based on the number of off-target site in an ascending order; 6) Map and extract the $10 \mathrm{bp}$ upstream and $4 \mathrm{bp}$ downstream flanking sequence of each selected gRNA, construct the TRAP target sequence as $10 \mathrm{bp}$ upstream $+23 \mathrm{bp}$ gRNA (include PAM) +4 bp downstream $=37$ bp; 7) Filter out TRAPs with BsmBI recognition site, because of GGA cloning; 8) Compared all the selected gRNAs with the database of CRISPR-iSTOP [56]; 9) Construct the full length sequence of each TRAP, which is $170 \mathrm{bp}$; In total, the first 12K TRAP-seq oligos contain 3832 genes and 12000 TRAPs were contained in the final TRAP-library. The 12K oligo pools was synthesized in Genscript ${ }^{\circledR}$ (Nanjing, China).

\section{TRAP-12K plasmid library preparation}

First, the TRAP $12 \mathrm{~K}$ oligos were cleaved and harvested from the microarray and diluted to $1 \mathrm{ng} / \mu \mathrm{L}$. Next, we performed PCR amplifications using the primers: TRAP-oligo (BsmBI GGA)-F: 5'TACAGCTaccacgtctcaCACC-3'; TRAP-oligo (BsmBI GGA)-R: 5'-AGCACAAccgtcgtctccAAAC3 .

The PCR reaction was carried out using PrimeSTAR HS DNA Polymerase (Takara, Japan) following the manufacturer's instruction. Briefly, each PCR reaction contained $1 \mu \mathrm{L}$ oligo template, $0.2 \mu \mathrm{L}$ PrimeSTAR polymerase, $1.6 \mu \mathrm{L}$ dNTP mixture, $4 \mu \mathrm{L}$ PrimeSTAR buffer, $1 \mu \mathrm{L}$ forward primer $(10$ $\mathrm{uM})$ and $1 \mu \mathrm{L}$ reverse primer $(10 \mathrm{uM})$ and $\mathrm{ddH} 2 \mathrm{O}$ to a final volume of $20 \mu \mathrm{L}$.

The thermocycle program was $98^{\circ} \mathrm{C} 2 \mathrm{~min},\left(98^{\circ} \mathrm{C} / 10 \mathrm{~s}, \quad 55^{\circ} \mathrm{C}^{*} / 10 \mathrm{~s}, \quad 72^{\circ} \mathrm{C} / 30 \mathrm{~s}\right)$ with $21 \mathrm{cycles}$, then $72^{\circ} \mathrm{C}$ for $7 \mathrm{~min}$ and $4^{\circ} \mathrm{C}$ hold. To avoid amplification bias of oligos introduced by PCR, we conducted 
685 686 687 688 689 690 691 692 693 694 695 696 697 698 699 700 701 702 703 704 705 706 707 708 709 710 711 712 713 714 715

gradient thermocycles and performed PCR products gray-intensity analysis to determine the optimal PCR cycles of 21 . The best thermocycles should be in the middle of an amplification curve. In this study, the PCR cycles was 21 for oligos amplification. But for PCR amplification of TRAP from cells integrated with TRAP lentivirus, the PCR cycle was 25 . The final TRAP PCR product length was $184 \mathrm{bp}$. We performed $72 \times$ parallel PCR reactions for $12 \mathrm{~K}$ oligos amplification, then these PCR products were pooled and gel purified by $2 \%$ agarose gel. $1 \mu \mathrm{g}$ purified PCR product were quantified with PCR-free next generation sequencing (MGI Tech).

The PCR products of TRAP oligos were then used for Golden Gate Assembly (GGA) to generate the TRAP 12K plasmids library. For each GGA reaction, the reaction mixture contained 100 ng pLentiTRAP-seq vector, $10 \mathrm{ng}$ purified 12K TRAP oligos-PCR products, $1 \mu \mathrm{L}$ T4 ligase (NEB), $2 \mu \mathrm{L}$ T4 ligase buffer (NEB), $1 \mu \mathrm{L}$ BsmBI restriction enzyme (ThermoFisher Scientific, FastDigestion) and $\mathrm{ddH} 2 \mathrm{O}$ to a final volume of $20 \mu \mathrm{L}$. The GGA reactions were performed at $37^{\circ} \mathrm{C} 5 \mathrm{~min}$ and $22^{\circ} \mathrm{C} 10$ min for 10 cycles, then $37^{\circ} \mathrm{C} 30 \mathrm{~min}$ and $75^{\circ} \mathrm{C} 15 \mathrm{~min} .36 \times$ parallel GGA reactions were performed and the ligation products were pooled into one tube.

Transformation was then carried out using home-made chemically competent DH5a cells. For each reaction, $10 \mu \mathrm{L}$ GGA ligation product was transformed in to $50 \mu \mathrm{L}$ competent cells and all the transformed cells were spread on one LB plate (15 cm dish in diameter) with Xgal, IPTG and Amp selection. High ligation efficiency was determined by the presence of very few blue colonies (also see Fig. S4). To ensure that there is sufficient coverage of each TRAP in the 12K TRAP-seq library, $42 \times$ parallel transformations were performed and all the bacterial colonies were scraped off and pooled together for plasmids midi-prep. For NGS-based quality quantification of TRAP coverage, midi-prep plasmids were used as DNA templates for TRAP PCR amplifications, followed by gel purification and NGS sequencing.

\section{TRAP-12K lentivirus packaging.}

HEK293T cells were used for lentivirus package. All the cells were cultured in Dulbecco's modified Eagle's medium (DMEM) (LONZA) supplemented with $10 \%$ fetal bovine serum (FBS) (Gibco), 1\% GlutaMAX (Gibco), and penicillin/streptomycin (100 units penicillin and $0.1 \mathrm{mg}$ streptomycin/mL) (The culture medium was named as D10 shortly) in a $37^{\circ} \mathrm{C}$ incubator with $5 \% \mathrm{CO} 2$ atmosphere and 
716

717

718

719

720

721

722

723

724

725

726

727

728

729

730

731

732

733

734

735

736

737

738

739

740

741

742

743

744

745

746

747

maximum humidity. Cells were passaged every 2-3 days when the confluence was approximately $80-$ $90 \%$.

For lentivirus packaging: Day 1: Wild-type HEK293T cells were seeded to a $10 \mathrm{~cm}$ culture dish, $4 \times$ $10^{6}$ cells per dish (10 dishes in total); Day 2: Transfection. Briefly, we refreshed the medium with 7 $\mathrm{mL}$ fresh culture medium to 1 hour before transfection (be gently, as the HEK293T cells are easy to be detached from the bottom of dish); Next, we performed transfection with the PEI 40000 transfection method. For $10 \mathrm{~cm}$ dish transfection, the DNA/PEI mixture contains $13 \mu \mathrm{g}$ pLentiTRAPseq $12 \mathrm{~K}$ vectors, $3 \mu \mathrm{g}$ pRSV-REV, $3.75 \mu \mathrm{g}$ pMD.2G, $13 \mu \mathrm{g}$ pMDGP-Lg/p-RRE, $100 \mu \mathrm{L}$ PEI 40000 solution $(1 \mu \mathrm{g} / \mu \mathrm{L}$ in sterilized $\mathrm{ddH} 2 \mathrm{O})$ and supplemented by serum-free opti MEM without phenol red (Invitrogen) to a final volume of $1 \mathrm{~mL}$. The transfection mixture was pipetted up and down several times gently, then kept at room temperature (RT) for $20 \mathrm{~min}$, then added into cells in a dropwise manner and mix by swirling gently. Day 3: Changed to fresh medium; Day 4: Harvest and filter all the culture medium of the $10 \mathrm{~cm}$ dish through a $0.45 \mu \mathrm{m}$ filter, pool the filtered media into one bottle. Each $10 \mathrm{~cm}$ dish generated approximately 7 8 $\mathrm{mL}$ lentivirus crude. Add polybrene solution (Sigma-Aldrich) in to the crude virus to a final concentration of $8 \mu \mathrm{g} / \mathrm{mL}$. Aliquot the crude virus into $15 \mathrm{~mL}$ tubes $(5 \mathrm{~mL} /$ tube $)$ and store in $-80{ }^{\circ} \mathrm{C}$ freezer.

\section{Lentivirus titer quantification by flow cytometry (FCM).}

As the pLenti-TRAP-seq vector expresses a EGFP gene, the functional titer of our lentivirus prep was assayed by FCM as described previously [57]. Briefly, 1) DAY 1: split and seed HEK293T cells to 24 -well plate, $5 \times 10^{4}$ cells per well. Generally, 18 wells were used to perform the titter detection, a gradient volume of the crude lentivirus was added into the cells and each volume was tested by replicates. In this experiment, the crude virus gradients were $2.5 \mu \mathrm{L}, 5 \mu \mathrm{L}, 10 \mu \mathrm{L}, 20 \mu \mathrm{L}, 40 \mu \mathrm{L}, 80$ $\mu \mathrm{L}, 160 \mu \mathrm{L}$ and $320 \mu \mathrm{L}$ for each well. Another 2 wells of cells were used for cell counting before transduction; 2) DAY 2: Conduct lentivirus transduction when cells reach up to $60 \sim 80 \%$ confluence. Before transduction, detach the last two wells of cells using $0.05 \%$ EDTA-Trypsin to determine the total number of cells in one well $\left(\mathrm{N}_{(\text {initial })}\right)$. Then change the remaining wells with fresh culture medium containing $8 \mu \mathrm{g} / \mathrm{mL}$ polybrene, then add the gradient volume of crude virus into each well and swirling gently to mix; 3) DAY 3: Change to fresh medium without polybrene; 4) DAY 4: Harvest all the cells and wash them twice in PBS. Fix the cells in 4\% formalin solution at RT for 20 min, then spin down the cell pellet at 2,000 rpm for $5 \mathrm{~min}$. Discard the supernatant and re-suspend the cell pellet 
carefully in $600 \mu \mathrm{L} \mathrm{PBS}$, and conduct FCM analysis immediately. FCM was performed using a BD LSRFortessa $^{\mathrm{TM}}$ cell analyzer with at least 30,000 events collected for each sample in replicates.

The FCM output data was analyzed by the software Flowjo vX.0.7. Percentage of GFP-positive cells was calculated as: $\mathcal{Y} \%=\mathrm{N}_{\text {(GFP-positive cells) }} / \mathrm{N}_{\text {(total cells) }} \times 100 \%$. Calculate the GFP percentage of all samples. For accurate titter determination, there should be a linear relationship between the GFP positive percentages and crude volume. The titter (Transducing Units (TU/mL) calculation according to this formula: $\mathrm{TU} / \mathrm{mL}=\left(\mathrm{N}_{\text {(initial) }} \times \mathcal{Y} \% \times 1000\right) / \mathrm{V}$. V represents the crude volume $(\mu \mathrm{L})$ used for initial transduction.

\section{Generation of Doxycycline-inducible spCas9/ABE7.10/CBE stable cell lines}

TRE-spCas9, TRE-ABE7.10 and TRE-CBE stable cells were generated by PiggyBac transposon systems. For stable cell lines establishment, HEK293T cells were transfected with pPB-TRE-spCas9Hygromycin (or pPB-ABE7.10-hygromycin, pPB-CBE-hygromycin) vector and pCMV-hybase with a 9:1 ratio. Briefly, $1 \times 10^{5}$ HEK293T cells were seeded in 24-well plate and transfections were conducted $24 \mathrm{~h}$ later using lipofectamine 2000 reagent following the manufacturer's instruction. Briefly, 450 ng pPB-TRE-spCas9-Hygromycin (or pPB-ABE7.10-hygromycin, pPB-CBEhygromycin) vectors and $50 \mathrm{ng}$ pCMV-hybase were mixed in $25 \mu \mathrm{L}$ optiMEM (tube A), then $1.5 \mu \mathrm{L}$ lipofectamine 2000 reagent was added in another $25 \mu \mathrm{L}$ optiMEM and mix gently (tube B). Incubate tube A and B at RT for 5 min, then add solution A into B gently and allow the mixture incubating at RT for $15 \mathrm{~min}$. Add the AB mixture into cells evenly in a dropwise manner. Cells transfected with pUC19 were acted as negative control. Culture medium was changed to selection medium with 50 $\mu \mathrm{g} / \mathrm{mL}$ hygromycin $48 \mathrm{~h}$ after transfection. Completion of selection took approximately 5-7 days until the negative cells were all dead in the un-transfected cells. The cells were allowed to grow in 50 $\mu \mathrm{g} / \mathrm{mL}$ hygromycin containing D10 medium for 3-5 days for further expansion. PCR-based

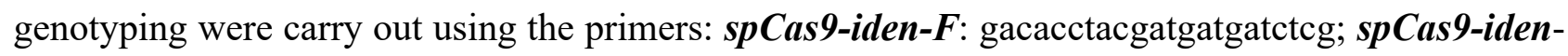
$\boldsymbol{R}$ : tggtgctcatcatagcgcttga; ABE7.10-iden-F: 5'-cagtactcgtgctcaacaatcg-3'; ABE7.10-iden-R: 5'ggcgttgcgaacaccgaataca-3';

BE4-iden-F: 5 '-ttcttcgatccgagagagctcc-3';

BE4-iden-R: 5'ctgcaccttgtgttcggacag-3'.

For functional tests of the spCas9, ABE7.10 and CBE4 expression cells, individual TRAP constructs packaged in lentivirus particles were transduced into the cells. Transduced cells were harvested for 
780

781

782

783

784

785

786

787

788

789

790

791

792

793

794

795

796

797

798

799

800

801

802

803

804

805

806

807

808

809

810

811

indel analysis 6 days after transduction. Indel analysis were carried out for both the TRAP site and the endogenous genome target sites.

\section{K TRAP-seq library lentivirus transduction}

HEK293T-SpCas9, -ABE7.10 and -CBE4 cells were cultured in D10 medium with $50 \mu \mathrm{g} / \mathrm{mL}$ hygromycin throughout the whole experiment. For 12K TRAP-seq library transduction, we followed the procedures showed in Fig. S7, S21, S31. Briefly, 1) Day -1: $2.5 \times 10^{6}$ cells per $10 \mathrm{~cm}$ dish were seeded, and 12 dishes in total. For each group, one dish was used for cell number determination before transduction and one dish for drug-resistance (puromycin) test control and the remaining 10 dishes were used for the $12 \mathrm{~K}$ TRAP-seq lentivirus library transduction; 2) Day 0: We determined the approximate cell number per dish by cell countering. This was used to determine the volume of crude lentivirus used for transduction using a multiplicity of infection (MOI) of 0.3 . The low MOI (0.3) ensures that most infected cells receive only 1 copy of the lentivirus construct with high probability [34]. The calculation formula is: $\mathrm{V}=\mathrm{N} \times 0.3 / \mathrm{TU} . \mathrm{V}=$ volume of lentivirus crude used for infection $(\mathrm{mL}) ; \mathrm{N}=$ cell number in the dish before infection; $\mathrm{TU}=$ the titter of lentivirus crude (IFU/mL). In this study, take the TRE-ABE7.10 group for instance, there were $1.875 \times 10^{7}$ cells in one dish, the TRAP $12 \mathrm{~K}$ lentivirus crude titter $=3.8 \times 10^{6} \mathrm{IFU} / \mathrm{mL}$. Thus $\mathrm{V}=1.875 \times 10^{7} \times 0.3 / 3.8 \times 10^{6}=$ $1.48 \mathrm{~mL}$. The $12 \mathrm{~K}$ TRAP-seq transduction coverage per dish is $1.875 \times 10^{7} \times 0.3 / 12000=469 \times$. As we performed 10 replicates for each group, the overall coverage would reach to about $4690 \times$. In this study, $\mathrm{V}_{\mathrm{spCas} 9}=1.26 \mathrm{~mL}, \mathrm{~V}_{\mathrm{ABE} 7.10}=1.48 \mathrm{~mL}, \mathrm{~V}_{\mathrm{wt}}=1.37 \mathrm{~mL}$ for each dish. For transduction, we added aforementioned volume of crude virus to each group in a dropwise manner and mix by swirling gently. The infected cells were cultured in a $37^{\circ} \mathrm{C}$ incubator; 3) Day 1: 24 hours after transduction, split the transduced cells of each dish to 3 dishes equally; 4) Day 2: For the 3 dishes of split (30 dishes in total, 3 divided into sub-groups), sub-group 1 (10 dishes) were harvested and labeled as the Day 2 after 12K TRAP-seq transduction. All cells from this sub-group were pooled into one tube and stored in $-20^{\circ} \mathrm{C}$ freezer for genomic DNA extraction; The sub-group 2 (10 dishes) was changed to fresh D10 medium contains $50 \mu \mathrm{g} / \mathrm{mL}$ hygromycin $+1 \mu \mathrm{g} / \mathrm{mL}$ puromycin (Dox-free group); The sub-group 3 (10 dishes) was changed to D10 medium contains $50 \mu \mathrm{g} / \mathrm{mL}$ hygromycin $+1 \mu \mathrm{g} / \mathrm{mL}$ puromycin +1 $\mu \mathrm{g} / \mathrm{mL}$ doxycycline (Dox-induction group). For the WT HEK293T cells (Group 3) screening, hygromycin but not puromycin should be excluded from the culture medium; 5) The transduced cells were spitted every 2 3 days when cell confluence reaches up to $90 \%$. At the indicated time points in Fig. S7, 21, 31, cells were harvested and stored in $-20^{\circ} \mathrm{C}$ freezer for further genomic DNA extraction. 


\section{PCR amplicons of TRAPs from cells}

814 Genomic DNA was extracted using the phenol-chloroform method. The genomic DNA were digested with RNase A (OMEGA) to remove RNA contamination (In this study, $50 \mu \mathrm{g}$ RNase A worked well to digest the RNA contamination in $100 \sim 200 \mu \mathrm{g}$ genomic DNA after incubating in $37^{\circ} \mathrm{C}$ for $30 \mathrm{~min}$ ). Then the genomic DNA was purified and subjected to PCR for amplification of the TRAP DNA. The PCR primers were: TRAP-NGS-F1: 5'-GGACTATCATATGCTTACCGTA-3' and TRAP-NGSR1: 5'-ACTCCTTTCAAGACCTAGCTAG-3'. The PCR product length is $252 \mathrm{bp}$. In this study, 5 $\mu \mathrm{g}$ genomic DNA was used as temperate in one PCR reaction which contained approximately $7.6 \times$ $10^{5}$ copies of TRAP construct (assuming $1 \times 10^{6}$ cells contain $6.6 \mu$ genomic DNA), which covered about $63 \times$ coverage of the $12 \mathrm{~K}$ TRAP-seq library. In total, $32 \times$ parallel PCR reactions were performed to achieve approximately $2,016 \times$ coverage of each TRAP construct. For each PCR reaction, briefly, $50 \mu \mathrm{L}$ PCR reaction system consists of $5 \mu \mathrm{g}$ genomic DNA, $0.5 \mu \mathrm{L}$ PrimeSTAR polymerase, $4 \mu \mathrm{L}$ dNTP mixture, $10 \mu \mathrm{L}$ PrimeSTAR buffer, $2.5 \mu \mathrm{L}$ forward primer $(10 \mathrm{uM})$ and 2.5 $\mu \mathrm{L}$ reverse primer $(10 \mathrm{uM})$ and supplemented with $\mathrm{ddH} 2 \mathrm{O}$ to a final volume of $50 \mu \mathrm{L}$. The thermocycle program was $98^{\circ} \mathrm{C} 2 \mathrm{~min},\left(98^{\circ} \mathrm{C}\right.$ for $10 \mathrm{~s}, \quad 55^{\circ} \mathrm{C}$ for $10 \mathrm{~s}, \quad 72^{\circ} \mathrm{C}$ for $\left.30 \mathrm{~s}\right)$ with 25 cycles, then $72^{\circ} \mathrm{C}$ for $7 \mathrm{~min}$ and $4^{\circ} \mathrm{C}$ hold. Then purify all the PCR products by $2 \%$ gel, pool the products together and conduct deep amplicon sequencing.

\section{Deep amplicon sequencing}

MGISEQ-500 (MGI of BGI in China) was used to perform the amplicons deep sequencing following the standard operation protocol. First, PCR-free library was prepared using MGIeasy FS PCR-free DNA library Prep kit following the manufacturer's instruction. Briefly, measure the concentration of purified PCR products using Qubit $4{ }^{\mathrm{TM}}$ fluorometer (Invitrogen) and dilute the concentration of each sample to $10 \mathrm{ng} / \mu \mathrm{L}$. $10 \mu \mathrm{L}$ diluted PCR product was mixed with an A-Tailing reaction which contained A-Tailing enzyme and buffer, incubated at $37^{\circ} \mathrm{C}$ for 30 minutes then $65^{\circ} \mathrm{C}$ for 15 min to inactive the enzyme. Then the A-Tailed sample was mixed with PCR Free index adapters (MGI.), T4 DNA Ligase and T4 ligase buffer to add index adapter at both 3' and 5' ends of PCR products. The reaction was incubated at $23^{\circ} \mathrm{C}$ for $30 \mathrm{~min}$ and then purified with XP beads. Then denature the PCR products to be single-strand DNA (ssDNA) by incubating at $95{ }^{\circ} \mathrm{C}$ for $3 \mathrm{~min}$ and keep on $4{ }^{\circ} \mathrm{C}$ for the 
844

845

846

847

848

849

850

851

852

853

854

855

856

857

858

859

860

861

862

863

864

865

866

867

868

869

870

871

872

873

874

875

by XP beads and assay the concentration of library by Qubit $4^{\mathrm{TM}}$ fluorometer. The amplicons libraries were subjected to deep sequencing on the MGISEQ-2000 platform. In this study, for each lane 4 samples (6 ng each) were pooled together for deep sequencing. To avoid sequencing bias induced by base unbalance of TRAP sequence, $12 \mathrm{ng}$ whole-genome DNA library (balance library) was mixed with the 4 PCR samples in a final concentration of $1.5 \mathrm{ng} / \mu \mathrm{L}$ and sequenced in one lane. All the samples were subjected to pair-ended $150 \mathrm{bp}$ deep-sequencing on MGISEQ-500 platform.

\section{Data analysis}

In order to evaluate the sequencing quality of amplicons and filter the low-quality sequencing data, the default parameters of Fastqc-0.11.3 (http://www.bioinformatics.babraham.ac.uk/projects/fastqc/) and fastp-0.19.6 (https://github.com/OpenGene/fastp) were used to carry out the filtration procedure and generate the clean dataset of each sample. The clean sequencing segments of pair-ended TRAP segments were merged using FLASh-1.2.1 (http://ccb.jhu.edu/software/FLASH/index.shtml) to obtain full-length TRAP constructs. The expression characteristics of all the sequences were analyzed by python 3.6, and most of the BsmBI linker fragments changed in orientation (GTTTGGAG-> GTTTGAAT). Therefore, in order to obtain the amplified fragment reads of each TRAP reference sequence, the TRAP sequence BsmBI Linker was removed from the reference sequence. The BWAMEM algorithm of bwa(http://bio-bwa.sourceforge.net/) was used for local alignment, and the reads of all samples were divided into 12,000 independent libraries. Due to the existence of sequencing or synthesis introduced errors, each library was then filtered. In order to simplify the filtering process, the filtration strategy varies from TRAP-ABE7.10, TRAP-CBE4.0-gam to TRAP-SpCas9. For ABE7.10 and CBE4.0-gam, they mainly cause single-base variation, rarely introduce insertion and deletion, the trap sequence length remains unchanged before and after editing. Therefore, we filter the sequence of each library by locking the intermediate $37 \mathrm{bp}$ sequence starting with gRNA + scaffold fragment and ending with GTTT. While TRAP-SpCas9 mainly cause insertion and deletion, the length of trap sequence change around $37 \mathrm{bp}$. Therefore, we adopt three steps to filter the sequence of each library. The first step is to obtain the sequence containing gRNA + scaffold fragment as dataset1.

The second step is to obtain the sequence containing GTTTGAAT in dataset1 as dataset2. The third step is to extract the intermediate trap sequence from dataset2, which removed the length limit. In order to eliminate the interference of background noise before analyzing editing efficiency, all mutations or indels found in WT HEK293T cells group were removed from the Dox group in advance. 
For the TRAP-ABE7.10 and TRAP-CBE4.0-gam, the total editing efficiency for each trap is calculated according to the following formula:

Total editing efficiency

$$
=\frac{\text { The number of reads whose trap sequence inconsistent with reference }}{\text { The total reads number of each trap }} \%
$$

, and the substitution percentage in the $37 \mathrm{bp}$ editing window is calculated according to the following formula:

Substitution percentage of $A B E$

$=\frac{\text { The number of reads with actual } A / T / C / G / N \text { base in specified position }}{\text { The total number of reads with theoretical } A \text { base in specified position }} \%$ Substitution percentage of $C B E$ $=\frac{\text { The number of reads with actual } A / T / C / G / N \text { base in specified position }}{\text { The total number of reads with theoretical } C \text { base in specified position }} \%$

For the TRAP-SpCas9 system, the total editing efficiency for each trap is calculated according to the following formula:

Total editing efficiency

$$
=\frac{\text { The number of reads whose trap length is not equal to } 37 \mathrm{bp}}{\text { The total reads number of each trap }} \%
$$

, and the average fraction of indels from $30 \mathrm{bp}$ deletion to $10 \mathrm{bp}$ insertion is calculated according to the following formula:

Average fraction of indels

$$
=\frac{\text { The number of reads whose trap length range from } 7-47 \mathrm{bp}}{\text { The total reads number of all } 12 \mathrm{k} \text { trap }} \%
$$

Example of selecting low and high efficency gRNA for ABE and CBE. At least one site in this sequence has more than $20 \%$ efficient for each TRAP, it is considered that the whole sequence has more than 20\% analytical value. Firstly, for the range of N1-N20, divide all TRAP library into a group with an efficiency of greater than $20 \%$ and others with an efficiency of $1 \%$, compare the base distribution of the two groups. Then for the range of N5-N7, divide all TRAP library into the above two groups, calculating the base mutation preference of $N-1 \& N+1$ sites, motif preference and the editing efficiency of N17-N20 sites including GCC, TT and other motif, respectively. For the CBE system, the statistical method is basically the same as that of ABE. Due to the high editing efficiency 
904

905

906

907

908

909

910

911

912

913

914

915

916

917

918

919

920

921

922

923

924

925

926

927

928

929

930

931

932

933

934

935

of $\mathrm{CBE}$, in order to correct statistical deviations, $\mathrm{CBE}$ divides the efficiency greater than $50 \%$ and less than 5\% into two groups when calculating the N1-N20 base preference and base mutation preference of $N-1 \& N+1$ site from N4-N8 . For the editing window is wider, the efficiency greater than $50 \%$ and less than $5 \%$ is divided into two groups during the motif preference in N4-N8 window and the editing efficiency of N17-N20 sites including GCC, TT and other motif . Python-3.6 and R scripts were used for efficiency and motif analysis of all the TRAP samples. All visualizations use GraphPad Prism8.2 and R package ggplot2.

\section{GNL machine learning featurization}

The feature set applied in our model construction contains 2485 features, which includes following five categories. (i) 604 features of "one-hot" encoding of the nucleotide. There are two subsets in this category: position-dependent and position-independent. And each category applies to the one nucleotide and pairwise nucleotide. Such as “_nuc_pd_Order2” consisted of e.g. AA_1/AT_1/AG_1, and “_nuc_pd_Order1" consisted of e.g. A_1/T_1/G_1/C_1. (ii) 3 GC features, which consists of GC count, GC count $<10$, GC count $>10$. (iii) 16 features of the two nucleotides flanking the NGG PAM in the 5' and 3'. (iv) Five thermodynamic features. We calculated five thermodynamic features ausing the "Tm_staluc function" in Biopython package. All these features above were derived from the 30mer of target sequences. (v) 1856 features of three nucleotides with "position-dependent" and "position-independent", such as ACG/AGG and ACG_1/ACT_2. Note that, all these features were encoded by 30 mer context sequence.(vi) Free energy (DeltaG), which was calculated by the local version of "mfold" (http://unafold.rna.albany.edu/?q=mfold/download-mfold). We used the binary programme "quikfold" in the same bin file of "mfold" to calculate multiple input sequences in the same time. All the parameters were set as default, to make sure the outputs of each 20 mer sequences be the same as the webpage.

\section{Model training: Comparison with other machine learning models}

We trained model to predict the cleavage efficiency of each site in the editing windows among ABE and CBE editing system. For the whole sequence sites of gRNA, three type of editing system was uniformly trained by the same BRR model. To select the optimized predictive model, we initially compared the predictive performance among eight models, they are, Bayesian Ridge regression (BRR), gradient boosted regression tree (GBRT), decision tree (DT), L1-regression (L1-reg), L2regression (L2-reg), linear regression (LR), neural network (NN), random forest (RF). All these 
algorithms were trained under the same features vector spaces. The mean performance of each algorithm was conducted by 10 -fold cross validation. Because all of them can be used as regression, the performance was evaluated by SCC (Spearman Correlation coefficient). The model with best performance, highest SCC value and lowest S.D., was selected. Finally, BRR was outperform than other counterparts. All the models applied for training using the scikit-learn package in python. During training, optimal hyper-parameter was chose using the inner 10-fold cross validation, using the grid search. After that, $\alpha$ and $\lambda$ were both set as 1.e- 6 .

\section{Bayesian Ridge Regression}

Bayesian Ridge regression is changed from Bayesian linear regression by adding the prior of coefficient " $\omega$ " as spherical Gaussian and the priors over lambda are chosen to be gamma distributions, which is similar to the classical Ridge regression [58]. Bayesian linear regression is briefly shown as (1), and the coefficients of $\mathrm{w}$ is hypothesized as the spherical distribution to find a maximum posteriori estimation of $\omega$ as (2) shows.

$$
\mathrm{P}(\mathrm{y} \mid \mathrm{X}, \omega, \alpha)=\mathrm{N}(\mathrm{y} \mid \mathrm{X} \omega, \alpha)
$$

Where $\alpha$ is treated as a random variable that is to be estimated from the data as gamma distribution.

$$
\mathrm{P}(\omega \mid \lambda)=\mathrm{N}\left(\omega \mid 0, \lambda^{-1} I_{p}\right)
$$

Where $\lambda$ is also treated as a random variable that is to be estimated from the data, and also be hypothesized as gamma distribution.

\section{Model explanation}

In addition to train the model with high performance, we also interested in the model importance for our final model. We used SHAP (SHapley Additive exPlanations) algorithm [59], which is a unified approach to explain the output of any machine learning model. Importantly, we excluded the necessary site when training the site model for each editing system. E.g. We drop A1 when training the N1 site of ABE system. So, the importance of the left features can be ranked by the SHAP value, and the top 20 important features of each model were shown for each editor.

\section{DATA AVAILABILITY}


965

966

967

968

969

970

971

972

973

974

975

976

977

978

979

980

981

982

983

984

985

986

987

988

989

990

991

992

993

994

995

996

997

998

999

NGS data: CNBG accession number: TBD

Code for machine learning: https://github.com/TerminatorJ/CRISPR-TRAP-seq.git

CRISPR atlas: www.crispratlas.com/crispr

\section{ACKNOWLEDGEMENT}

This project is supported by the Sanming Project of Medicine in Shenzhen (SZSM201612074), Qingdao-Europe Advanced Institute for Life Sciences Grant, Guangdong Provincial Key Laboratory of Genome Read and Write (No. 2017B030301011) and Guangdong Provincial Academician Workstation of BGI Synthetic Genomics (No. 2017B090904014) . Y.L. is supported by BGIResearch and Brainstem Center of Excellence (Danish Innovation Fund, BrainStem). L.L. is supported by the Lundbeck Foundation (R219-2016-1375) and the DFF Sapere Aude Starting grant (8048-00072A). G.C. is supported by National Human Genome Research Institute of the National Institutes of Health [RM1HG008525]. We thank the China National GeneBank for the support of executing the project under the framework of Genome Read and Write.

\section{AUTHOR CONTRIBUTION}

L.L. and Y.L. conceived the idea. L.B, G.C, L.L and Y.L oversaw the whole study. X.X, K.Q, L.L. and Y.L. and designed the study. X.X, K.Q, X.L, and X.P performed most of the experiments and analyses. All authors have contributed to the execution of the experiments and studies. L.L. and Y.L. drafted the manuscript. All authors discussed the results and contributed to the final manuscript.

\section{CONFLICT OF INTEREST}

The authors declare no conflict of interest.

\section{REFERENCES}

1. Mali, P., et al., RNA-guided human genome engineering via Cas9. Science, 2013. 339(6121):

2. Cong, L., et al., Multiplex genome engineering using CRISPR/Cas systems. Science, 2013. 339(6121): p. 819-23.

3. Jinek, M., et al., A programmable dual-RNA-guided DNA endonuclease in adaptive bacterial immunity. Science, 2012. 337(6096): p. 816-21.

4. Farboud, B., et al., Enhanced Genome Editing with Cas9 Ribonucleoprotein in Diverse Cells and Organisms. J Vis Exp, 2018(135).

5. Hwang, W.Y., et al., Efficient genome editing in zebrafish using a CRISPR-Cas system. Nat Biotechnol, 2013. 31(3): p. 227-9. 
1000 6. Friedland, A.E., et al., Characterization of Staphylococcus aureus Cas9: a smaller Cas9 for all-

1001

1002

1003

1004

1005

1006

1007

1008

1009

1010

1011

1012

1013

1014

1015

1016

1017

1018

1019

1020

1021

1022

1023

1024

1025

1026

1027

1028

1029

1030

1031

1032

1033

1034

1035

1036

1037

1038

1039

1040

1041

1042

1043

1044

in-one adeno-associated virus delivery and paired nickase applications. Genome Biol, 2015. 16: p. 257.

7. Muller, M., et al., Streptococcus thermophilus CRISPR-Cas9 Systems Enable Specific Editing of the Human Genome. Mol Ther, 2016. 24(3): p. 636-44.

8. Hou, Z., et al., Efficient genome engineering in human pluripotent stem cells using Cas 9 from Neisseria meningitidis. Proc Natl Acad Sci U S A, 2013. 110(39): p. 15644-9.

9. Kleinstiver, B.P., et al., Broadening the targeting range of Staphylococcus aureus CRISPRCas9 by modifying PAM recognition. Nat Biotechnol, 2015. 33(12): p. 1293-1298.

10. Zetsche, B., et al., Cpf1 is a single RNA-guided endonuclease of a class 2 CRISPR-Cas system. Cell, 2015. 163(3): p. 759-71.

11. Cox, D.B.T., et al., RNA editing with CRISPR-Cas13. Science, 2017. 358(6366): p. 1019-1027.

12. Yeh, C.D., C.D. Richardson, and J.E. Corn, Advances in genome editing through control of DNA repair pathways. Nat Cell Biol, 2019. 21(12): p. 1468-1478.

13. Qi, L.S., et al., Repurposing CRISPR as an RNA-guided platform for sequence-specific control of gene expression. Cell, 2013. 152(5): p. 1173-83.

14. Lin, L., et al., Genome-wide determination of on-target and off-target characteristics for RNAguided DNA methylation by dCas9 methyltransferases. Gigascience, 2018. 7(3): p. 1-19.

15. Chen, B., J. Guan, and B. Huang, Imaging Specific Genomic DNA in Living Cells. Annu Rev Biophys, 2016. 45: p. 1-23.

16. Kang, B.C., et al., Precision genome engineering through adenine base editing in plants. Nat Plants, 2018. 4(7): p. 427-431.

17. Zong, Y., et al., Precise base editing in rice, wheat and maize with a Cas9-cytidine deaminase fusion. Nat Biotechnol, 2017. 35(5): p. 438-440.

18. Komor, A.C., et al., Programmable editing of a target base in genomic DNA without doublestranded DNA cleavage. Nature, 2016. 533(7603): p. 420-4.

19. Gaudelli, N.M., et al., Programmable base editing of $A^{*} T$ to $G^{*} C$ in genomic DNA without DNA cleavage. Nature, 2017. 551(7681): p. 464-471.

20. Koblan, L.W., et al., Improving cytidine and adenine base editors by expression optimization and ancestral reconstruction. Nat Biotechnol, 2018. 36(9): p. 843-846.

21. Rees, H.A., et al., Improving the DNA specificity and applicability of base editing through protein engineering and protein delivery. Nat Commun, 2017. 8: p. 15790.

22. Nishida, K., et al., Targeted nucleotide editing using hybrid prokaryotic and vertebrate adaptive immune systems. Science, 2016. 353(6305).

23. Zhang, Y., et al., Programmable base editing of zebrafish genome using a modified CRISPRCas9 system. Nat Commun, 2017. 8(1): p. 118.

24. Jensen, K.T., et al., Chromatin accessibility and guide sequence secondary structure affect CRISPR-Cas9 gene editing efficiency. FEBS Lett, 2017. 591(13): p. 1892-1901.

25. Uusi-Makela, M.I.E., et al., Chromatin accessibility is associated with CRISPR-Cas9 efficiency in the zebrafish (Danio rerio). PLoS One, 2018. 13(4): p. e0196238.

26. Kim, H.K., et al., High-throughput analysis of the activities of xCas9, SpCas9-NG and SpCas9 at matched and mismatched target sequences in human cells. Nat Biomed Eng, 2020. 4(1): p. 111-124.

27. Shen, M.W., et al., Predictable and precise template-free CRISPR editing of pathogenic variants. Nature, 2018. 563(7733): p. 646-651. 
1045

1046

1047

1048

1049

1050

1051

1052

1053

1054

1055

1056

1057

1058

1059

1060

1061

1062

1063

1064

1065

1066

1067

1068

1069

1070

1071

1072

1073

1074

1075

1076

1077

1078

1079

1080

1081

1082

1083

1084

1085

1086

1087

1088

28. Allen, F., et al., Predicting the mutations generated by repair of Cas9-induced double-strand breaks. Nat Biotechnol, 2018.

29. Zhou, Y., et al., Enhanced genome editing in mammalian cells with a modified dualfluorescent surrogate system. Cell Mol Life Sci, 2016. 73(13): p. 2543-63.

30. Komor, A.C., et al., Improved base excision repair inhibition and bacteriophage Mu Gam protein yields C:G-to-T:A base editors with higher efficiency and product purity. Sci Adv, 2017. 3(8): p. eaao4774.

31. Sjostedt, E., et al., An atlas of the protein-coding genes in the human, pig, and mouse brain. Science, 2020. 367(6482).

32. Billon, P., et al., CRISPR-Mediated Base Editing Enables Efficient Disruption of Eukaryotic Genes through Induction of STOP Codons. Mol Cell, 2017. 67(6): p. 1068-1079 e4.

33. $\mathrm{Xu}, \mathrm{Y}$., et al., A new massively parallel nanoball sequencing platform for whole exome research. BMC Bioinformatics, 2019. 20(1): p. 153.

34. Shalem, O., et al., Genome-scale CRISPR-Cas9 knockout screening in human cells. Science, 2014. 343(6166): p. 84-87.

35. Morgens, D.W., et al., Genome-scale measurement of off-target activity using Cas9 toxicity in high-throughput screens. Nat Commun, 2017. 8: p. 15178.

36. Tycko, J., V.E. Myer, and P.D. Hsu, Methods for Optimizing CRISPR-Cas9 Genome Editing Specificity. Mol Cell, 2016. 63(3): p. 355-70.

37. Kosicki, M., et al., Dynamics of Indel Profiles Induced by Various CRISPR/Cas9 Delivery Methods. Prog Mol Biol Transl Sci, 2017. 152: p. 49-67.

38. Lin, L. and Y. Luo, Tracking CRISPR's Footprints. Methods Mol Biol, 2019. 1961: p. 13-28.

39. Moller, H.D., et al., CRISPR-C: circularization of genes and chromosome by CRISPR in human cells. Nucleic Acids Res, 2018. 46(22): p. e131.

40. Wang, D., et al., Optimized CRISPR guide RNA design for two high-fidelity Cas9 variants by deep learning. Nat Commun, 2019. 10(1): p. 4284.

41. Graf, R., et al., sgRNA Sequence Motifs Blocking Efficient CRISPR/Cas9-Mediated Gene Editing. Cell Rep, 2019. 26(5): p. 1098-1103 e3.

42. Ata, H., et al., Robust activation of microhomology-mediated end joining for precision gene editing applications. PLoS Genet, 2018. 14(9): p. e1007652.

43. Wang, J., et al., GNL-Scorer: A generalized model for predicting CRISPR on-target activity by machine learning and featurization. J Mol Cell Biol, 2020.

44. Concordet, J.P. and M. Haeussler, CRISPOR: intuitive guide selection for CRISPR/Cas9 genome editing experiments and screens. Nucleic Acids Res, 2018. 46(W1): p. W242-W245.

45. Xue, L., et al., Prediction of CRISPR sgRNA Activity Using a Deep Convolutional Neural Network. J Chem Inf Model, 2019. 59(1): p. 615-624.

46. Labuhn, M., et al., Refined sgRNA efficacy prediction improves large- and small-scale CRISPRCas9 applications. Nucleic Acids Res, 2018. 46(3): p. 1375-1385.

47. Hwang, G.H., et al., Web-based design and analysis tools for CRISPR base editing. BMC Bioinformatics, 2018. 19(1): p. 542.

48. Dandage, R., et al., beditor: A Computational Workflow for Designing Libraries of Guide RNAs for CRISPR-Mediated Base Editing. Genetics, 2019. 212(2): p. 377-385.

49. Ramakrishna, S., et al., Surrogate reporter-based enrichment of cells containing RNA-guided Cas9 nuclease-induced mutations. Nat Commun, 2014. 5: p. 3378. 
1089

1090

1091

1092

1093

1094

1095

1096

1097

1098

1099

1100

1101

1102

1103

1104

1105

1106

1107

1108

1109

1110

50. Kim, H., et al., Surrogate reporters for enrichment of cells with nuclease-induced mutations. Nat Methods, 2011. 8(11): p. 941-3.

51. Cullot, G., et al., CRISPR-Cas9 genome editing induces megabase-scale chromosomal truncations. Nat Commun, 2019. 10(1): p. 1136.

52. Kosicki, M., K. Tomberg, and A. Bradley, Repair of double-strand breaks induced by CRISPRCas9 leads to large deletions and complex rearrangements. Nat Biotechnol, 2018. 36(8): p. 765-771.

53. Hu, J.H., et al., Evolved Cas9 variants with broad PAM compatibility and high DNA specificity. Nature, 2018. 556(7699): p. 57-63.

54. Slaymaker, I.M., et al., Rationally engineered Cas9 nucleases with improved specificity. Science, 2016. 351(6268): p. 84-8.

55. Kleinstiver, B.P., et al., High-fidelity CRISPR-Cas9 nucleases with no detectable genome-wide off-target effects. Nature, 2016. 529(7587): p. 490-5.

56. Kuscu, C., et al., CRISPR-STOP: gene silencing through base-editing-induced nonsense mutations. Nat Methods, 2017. 14(7): p. 710-712.

57. Kutner, R.H., X.Y. Zhang, and J. Reiser, Production, concentration and titration of pseudotyped HIV-1-based lentiviral vectors. Nat Protoc, 2009. 4(4): p. 495-505.

58. Tipping, M.E., Sparse Bayesian learning and the relevance vector machine. Journal of machine learning research, 2001. 1(Jun): p. 211-244.

59. Lundberg, S.M. and S.-I. Lee. A unified approach to interpreting model predictions. 2017. 